\title{
Integrable nonlinear evolution equations on a finite interval
}

\author{
A. Boutet de Monvel \\ Institut de Mathématiques de Jussieu, case 7012, Université Paris 7, \\ 2 place Jussieu, 75251 Paris, France \\ A.S. Fokas \\ Department of Applied Mathematics and Theoretical Physics, University of Cambridge, \\ Cambridge CB3 0WA, U.K. \\ D. Shepelsky \\ Mathematical Division, Institute for Low Temperature Physics, \\ 47 Lenin Avenue, 61103 Kharkiv, Ukraine
}

August 9, 2005

\begin{abstract}
Let $q(x, t)$ satisfy an integrable nonlinear evolution PDE on the interval $0<x<L$, and let the order of the highest $x$-derivative be $n$. For a problem to be at least linearly well-posed one must prescribe $N$ boundary conditions at $x=0$ and $n-N$ boundary conditions at $x=L$, where if $n$ is even, $N=n / 2$, and if $n$ is odd, $N$ is either $(n-1) / 2$ or $(n+1) / 2$, depending on the sign of $\partial_{x}^{n} q$. For example, for the sine-Gordon ( $\mathrm{sG}$ ) equation one must prescribe one boundary condition at each end, while for the modified Korteweg-de Vries $(\mathrm{mKdV})$ equations involving $q_{t}+q_{x x x}$ and $q_{t}-q_{x x x}$ one must prescribe one and two boundary conditions, respectively, at $x=0$. We will refer to these two $\mathrm{mKdV}$ equations as $\mathrm{mKdV}$ I and $\mathrm{mKdV}$ II, respectively.

Here we analyze the Dirichlet problem for the sG equation, as well as typical boundary value problems for the $\mathrm{mKdV}$ I and $\mathrm{mKdV}$ II equations. We first show that the unknown boundary values at each end (for example, $q_{x}(0, t)$ and $q_{x}(L, t)$ in the case of the Dirichlet problem for the $\mathrm{sG}$ equation) can be expressed in terms of the given initial and boundary conditions through a system of four nonlinear ODEs. For the sG and the focusing versions of $\mathrm{mKdV}$ I and $\mathrm{mKdV}$ II equations, this system has a global solution, while for the defocusing versions of mKdV I and mKdV II equations, the global existence remains open. We then show that $q(x, t)$ can be expressed in terms of the solution of a $2 \times 2$ matrix Riemann-Hilbert problem formulated in the complex $k$-plane. This problem has explicit $(x, t)$ dependence in the form of an exponential; for example, for the case of the $\mathrm{sG}$ this exponential is $\exp \{i(k-1 / k) x+i(k+1 / k) t\}$. Furthermore, the relevant jump matrices are explicitly given in terms of the spectral functions $\{a(k), b(k)\},\{A(k), B(k)\}$, and $\{\mathcal{A}(k), \mathcal{B}(k)\}$, which in turn are defined in terms of the initial conditions, of the boundary values of $q$ and of its $x$-derivatives at $x=0$, and of the boundary values of $q$ and of its $x$-derivatives at $x=L$, respectively. This Riemann-Hilbert problem has a global solution.
\end{abstract}

\section{Introduction}

The sine-Gordon (sG), the modified Korteweg-de Vries I (mKdV I) and the modified Korteweg-de Vries II (mKdV II) equations are examples of integrable nonlinear evolution equations in one space variable. Integrable PDEs have the distinctive property that they can be written as the compatibility condition of two linear eigenvalue equations, which are called a Lax pair [1]. An effective method for solving the initial value problem for integrable evolution equations on the line was discovered in 1967 [2]. This method can be thought of as a nonlinear Fourier transform method. It was realized in [3] that the extension of this method to initial boundary value problems requires a deeper understanding of the following question: What is the fundamental transform for solving initial boundary value problems for linear evolution equations with $x$-derivatives of arbitrary order? The investigation of this question has led to the discovery of a 
general approach for solving boundary value problems for linear and for integrable nonlinear PDEs [4]. For integrable nonlinear evolution PDEs this approach is based on the simultaneous spectral analysis of the two linear eigenvalue equations forming the Lax pair, and on the investigation of the so-called global relation, which is an algebraic relation coupling the relevant spectral functions.

Regarding initial boundary value problems for nonlinear integrable evolution equations on the half-line we note the following: The rigorous implementation of the method of [3] to the nonlinear Schrödinger equation (NLS) was presented in [5]. Analogous results for the sG, the KdV (with dominant surface tension) and the mKdV II equations were presented in [6] and [7]. The most difficult step in the method of [3] is the analysis of the global relation. Although rigorous results in this direction were obtained in [5], the relevant formalism is quite complicated. A dramatic simplification was announced in [8] where it was shown that the global relation can be effectively analyzed if one introduces a Gelfand-LevitanMarchenko representation for the eigenfunction of the $t$-part of the Lax pair evaluated at $x=0$. If this eigenfunction is denoted by $\left(\Phi_{1}(t, k), \Phi_{2}(t, k)\right)^{T}$, and if the functions involved in its Gelfand-LevitanMarchenko representation are denoted by $\left\{\hat{L}_{j}, \hat{M}_{j}\right\}_{1}^{2}$, then it is shown in [8] that, in the case of the Dirichlet problem for the NLS equation, $q_{x}(0, t)$ can be explicitly expressed in terms of $\left\{\hat{L}_{j}, \hat{M}_{j}\right\}_{1}^{2}$ and of the initial and boundary conditions $(q(x, 0)$ and $q(0, t))$. This yields $q_{x}(0, t)$ in terms of a system of four nonlinear ODEs satisfied by the functions $\left\{\hat{L}_{j}, \hat{M}_{j}\right\}_{1}^{2}$. A further important development is presented in [9], where it is shown that it is possible to express $\left\{\hat{L}_{j}, \hat{M}_{j}\right\}_{1}^{2}$ in terms of $\Phi_{1}$ and $\Phi_{2}$. Thus the formalism presented in [9] expresses $q_{x}(0, t)$ in terms of a system of two nonlinear ODEs satisfied by the functions $\Phi_{1}$ and $\Phi_{2}$. Similarly, it is shown in [9] that the unknown boundary values for the sG, the mKdV I, and the mKdV II equations can also be expressed in terms of a system of two nonlinear ODEs. Furthermore, it is shown in [9] that this system for the sG and for the focusing versions of the NLS,the mKdV I, and the mKdV II equations has a global solution.

Regarding initial boundary value problems for nonlinear integrable evolution equations on the interval we note the following: The solution $q(x, t)$ of the mKdV II equation in terms of a $2 \times 2$ matrix RiemannHilbert problem was obtained in [10]. However, the relevant global relation was not analyzed in this paper. The analogous RH problem for the NLS equation together with the analysis of the global relation is presented in [11]; the latter analysis is based on the results of [8].

In the present paper, using the general methodology of [6] and the recent results of [9], we analyze the following IBV problems:

- $s \mathrm{G}$

$$
\begin{array}{cc}
\frac{\partial^{2} q}{\partial t^{2}}-\frac{\partial^{2} q}{\partial x^{2}}+\sin q=0, \quad 0<x<L, & 0<t<T, \\
q(x, 0)=q_{0}(x), \quad q_{t}(x, 0)=q_{1}(x), & 0<x<L, \\
q(0, t)=g_{0}(t), \quad q(L, t)=h_{0}(t), & 0<t<T
\end{array}
$$

- MKdV I

$$
\begin{aligned}
& \frac{\partial q}{\partial t}+\frac{\partial^{3} q}{\partial x^{3}}-6 \lambda q^{2} q_{x}=0, \quad \lambda= \pm 1, \quad 0<x<L, \quad 0<t<T \\
& \begin{aligned}
q(x, 0)=q_{0}(x), \quad 0<x<L, \\
q(0, t)=g_{0}(t), \quad q(L, t)=h_{0}(t), \quad q_{x}(L, t)=h_{1}(t), \quad 0<t<T
\end{aligned}
\end{aligned}
$$

- $\mathrm{mKdV}$ II

$$
\begin{aligned}
& \frac{\partial q}{\partial t}-\frac{\partial^{3} q}{\partial x^{3}}+6 \lambda q^{2} q_{x}=0, \quad \lambda= \pm 1, \quad 0<x<L, \quad 0<t<T \\
& \begin{aligned}
q(x, 0)=q_{0}(x), \quad 0<x<L, \\
q(0, t)=g_{0}(t), \quad q_{x}(0, t)=g_{1}(t), \quad q(L, t)=h_{0}(t), \quad 0<t<T
\end{aligned}
\end{aligned}
$$

We assume that $q(x, t)$ is a real-valued function and that the functions $q_{0}(x), q_{1}(x), g_{j}(t), h_{j}(t)$ are sufficiently smooth. We also assume that the given initial and boundary values are compatible at $\{x=0, t=0\}$ and at $\{x=L, t=0\}$. 
The above equations admit a Lax pair formulation of the form

$$
\begin{aligned}
\mu_{x}+i f_{1}(k) \hat{\sigma}_{3} \mu & =Q(x, t, k) \mu, \\
\mu_{t}+i f_{2}(k) \hat{\sigma}_{3} \mu & =\tilde{Q}(x, t, k) \mu, \quad k \in \mathbb{C} .
\end{aligned}
$$

Here $\hat{\sigma}$ denotes the matrix commutator with the Pauli matrix $\sigma_{3}$ :

$$
\sigma_{3}=\operatorname{diag}\{1,-1\}, \quad \hat{\sigma}_{3} A=\left[\sigma_{3}, A\right], \quad e^{\hat{\sigma}_{3}} A=e^{\sigma_{3}} A e^{-\sigma_{3}},
$$

for any $2 \times 2$ matrix $A$.

The eigenfunction $\mu(x, t, k)$ is a $2 \times 2$ matrix valued function of the arguments indicated, $f_{1}(k)$ and $f_{2}(k)$ are given analytic functions of $k$, and the $2 \times 2$ matrix valued functions $Q$ and $\tilde{Q}$ are given functions of $k$, of $q(x, t)$, and of the derivatives of $q(x, t)$. The particular form of these functions for the $\mathrm{s}$, the mKdV I, and the mKdV II equations will be given in Section 2.

The analysis of an initial boundary value problem for an equation possessing the Lax pair (1.7) involves the following steps.

\section{Step 1: A Riemann-Hilbert formulation under the assumption of existence.}

We assume that there exists a smooth solution $q(x, t), 0<x<L, 0<t<T$.

We use the simultaneous spectral analysis of the associated Lax pair to express $q(x, t)$ in terms of the solution of a $2 \times 2$-matrix Riemann- Hilbert $(\mathrm{RH})$ problem defined in the complex $k$-plane. This problem has explicit $(x, t)$ dependence in the form of $\exp \left\{2 i\left(f_{1}(k) x+f_{2}(k) t\right)\right\}$, and it is uniquely defined in terms of the so-called spectral functions,

$$
\{a(k), b(k)\}, \quad\{A(k), B(k)\}, \quad\{\mathcal{A}(k), \mathcal{B}(k)\} .
$$

The spectral functions $\{a(k), b(k)\}$ are expressed in terms of the initial conditions, while $\{A(k), B(k)\}$ and $\{\mathcal{A}(k), \mathcal{B}(k)\}$ are expressed in terms of the boundary values $\left\{g_{l}(t)\right\}_{0}^{n-1}$ and $\left\{h_{l}(t)\right\}_{0}^{n-1}$, respectively, where $g_{l}(t)=\partial_{x}^{l} q(0, t)$ and $h_{l}(t)=\partial_{x}^{l} q(L, t)$. For the $\mathrm{sG}$ equation, $n=2$, while for the $\mathrm{mKdV} \mathrm{I}$ and mKdV II equations, $n=3$.

Furthermore, we will show that the spectral functions (1.8) are not independent but they satisfy the global relation

$$
e^{2 i f_{1}(k) L}(\overline{a(\bar{k})} A(k)-\rho \overline{b(\bar{k})} B(k)) \mathcal{B}(k)-(a(k) B(k)-b(k) A(k)) \mathcal{A}(k)=e^{2 i f_{2}(k) T} c(k),
$$

where $c(k)=c(k ; T)$ is of $O\left(\frac{1+e^{2 i f_{1}(k) L}}{k}\right)$ as $|k| \rightarrow \infty$ and

$$
\rho= \begin{cases}\lambda & \text { for } \mathrm{mKdV} \text { I and II } \\ -1 & \text { for } \mathrm{sG}\end{cases}
$$

Step 2: Existence under the assumption that the spectral functions satisfy the global relation.

Motivated from the results of Step 1, we define the spectral functions (1.8) in terms of the initial conditions and in terms of the smooth functions $\left\{g_{l}(t)\right\}_{0}^{n-1}$ and $\left\{h_{l}(t)\right\}_{0}^{n-1}$. We assume that the boundary values are such that the spectral functions satisfy the global relation (1.9). We also define $q(x, t)$ in terms of the solution of the RH problem formulated in Step 1. We then prove that: (i) $q(x, t)$ is defined globally for all $0<x<L, 0<t<T$; (ii) $q(x, t)$ solves the given nonlinear equation; (iii) $q(x, t)$ satisfies the initial conditions; furthermore, $\partial_{x}^{l} q(0, t)=g_{l}(t), \partial_{x}^{l} q(L, t)=h_{l}(t), 0 \leq l \leq n-1$.

\section{Step 3: The analysis of the global relation}

Given a subset of the boundary values $\left\{g_{l}(t)\right\}_{0}^{n-1}$ and $\left\{h_{l}(t)\right\}_{0}^{n-1}$ as boundary conditions $(N$ boundary conditions at $x=0$ and $n-N$ boundary conditions at $x=L$ ), we characterize the remaining part of the boundary values through the solution of a system of nonlinear ODEs. For the sG and the $\mathrm{mKdV} \mathrm{I}$ equations, $N=1$, while for the $\mathrm{mKdV}$ II equations, $N=2$. In certain cases this system can be shown to have a global solution. 


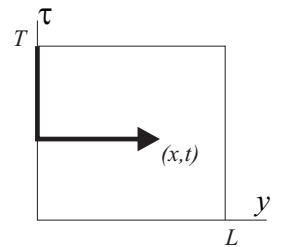

$\mu_{1}$

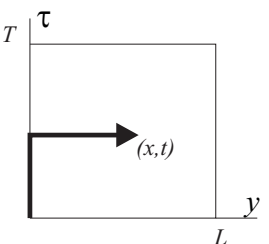

$\mu_{2}$

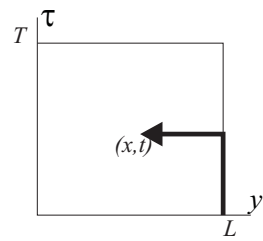

$\mu_{3}$

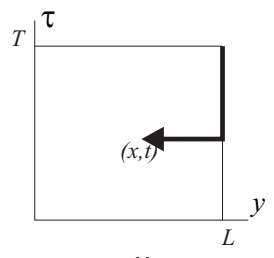

$\mu_{4}$

Figure 1: The contours used for the definition of $\mu_{j}, j=1, \ldots, 4$

We now discuss further the above steps.

The analysis of Step 1 is based on the introduction of appropriate eigenfunctions which satisfy both parts of the Lax pair. It was shown in [12] that for a polygonal domain with $N$ corners, there exists a canonical way of choosing such eigenfunctions: there exist $N$ such eigenfunctions, each of them normalized with respect to each corner. Thus in our case we introduce four eigenfunctions, $\left\{\mu_{j}(x, t, k)\right\}_{1}^{4}$, see Figure 1 , such that

$$
\mu_{1}(0, T, k)=I, \quad \mu_{2}(0,0, k)=I, \quad \mu_{3}(L, 0, k)=I, \quad \mu_{4}(L, T, k)=I,
$$

where $\mu_{j}$ are $2 \times 2$ matrices and $I=\operatorname{diag}\{1,1\}$.

It can be shown that these eigenfunctions are simply related through three matrices $s, S$, and $S_{L}$,

$$
\begin{aligned}
s(k) & =\mu_{3}(0,0, k), \quad S(k)=\left(e^{i f_{2}(k) T \sigma_{3}} \mu_{2}(0, T, k) e^{-i f_{2}(k) T \sigma_{3}}\right)^{-1}, \\
S_{L}(k) & =\left(e^{i f_{2}(k) T \sigma_{3}} \mu_{3}(L, T, k) e^{-i f_{2}(k) T \sigma_{3}}\right)^{-1} .
\end{aligned}
$$

Theses matrices satisfy certain symmetry properties, thus they can be denoted by

$$
s(k)=\left(\begin{array}{cc}
\overline{a(\bar{k})} & b(k) \\
\rho \overline{b(\bar{k})} & a(k)
\end{array}\right), \quad S(k)=\left(\begin{array}{cc}
\overline{A(\bar{k})} & B(k) \\
\rho \overline{B(\bar{k})} & A(k)
\end{array}\right), \quad S_{L}(k)=\left(\begin{array}{cc}
\overline{\mathcal{A}(\bar{k})} & \mathcal{B}(k) \\
\rho \overline{\mathcal{B}(\bar{k})} & \mathcal{A}(k)
\end{array}\right) .
$$

Regarding Step 2 we note that equations (1.11) and (1.12) motivate the definitions

$$
\begin{aligned}
& a(k)=\varphi_{2}(0, k), \quad b(k)=\varphi_{1}(0, k), \\
& A(k)=\overline{\Phi_{2}(T, \bar{k})}, \quad B(k)=-e^{2 i f_{2}(k) T} \Phi_{1}(T, k), \\
& \mathcal{A}(k)=\overline{\Psi_{2}(T, \bar{k})}, \quad \mathcal{B}(k)=-e^{2 i f_{2}(k) T} \Psi_{1}(T, k), \quad k \in \mathbb{C},
\end{aligned}
$$

where the vectors

$$
\left(\varphi_{1}(x, k), \varphi_{2}(x, k)\right)^{T}, \quad\left(\Phi_{1}(t, k), \Phi_{2}(t, k)\right)^{T}, \quad\left(\Psi_{1}(t, k), \Psi_{2}(t, k)\right)^{T}
$$

satisfy the boundary conditions

$$
\left(\begin{array}{l}
\varphi_{1}(L, k) \\
\varphi_{2}(L, k)
\end{array}\right)=\left(\begin{array}{l}
0 \\
1
\end{array}\right), \quad\left(\begin{array}{l}
\Phi_{1}(0, k) \\
\Phi_{2}(0, k)
\end{array}\right)=\left(\begin{array}{l}
0 \\
1
\end{array}\right), \quad\left(\begin{array}{l}
\Psi_{1}(0, k) \\
\Psi_{2}(0, k)
\end{array}\right)=\left(\begin{array}{l}
0 \\
1
\end{array}\right)
$$

and they solve the $x$-part of the Lax pair evaluated at $t=0$, the $t$-part of the Lax pair evaluated at $x=0$, and the $t$-part of the Lax pair evaluated at $x=L$, respectively. Thus the vectors (1.14) depend on the initial conditions, on the boundary values at $x=0,\left\{g_{l}(t)\right\}_{0}^{n-1}$, and on the boundary values at $x=L,\left\{h_{l}(t)\right\}_{0}^{n-1}$, respectively.

The global existence of $q(x, t)$ is based on the unique solvability of the associated RH problem, which in turn is based on the distinctive nature of the functions defining the jump matrices: the functions have explicit $(x, t)$ dependence in an exponential form and they involve the spectral functions $s(k), S(k)$, and $S_{L}(k)$, which have the symmetry properties expressed in (1.12). Using theses facts it can be shown that the associated homogeneous $\mathrm{RH}$ problem has only the trivial solution (i.e. there exists a vanishing 
lemma). The proof that $q(x, t)$ solves the given nonlinear PDE uses the standard arguments of the dressing method [15]. The proof that $q(x, 0)=q_{0}(x)\left(q(x, 0)=q_{0}(x)\right.$ and $q_{t}(x, 0)=q_{1}(x)$ in the case of the $\mathrm{SG}$ ) is based on the fact that the $\mathrm{RH}$ problem satisfied at $t=0$ is equivalent to a $\mathrm{RH}$ problem derived in terms of $s(k)$ which characterizes $q_{0}(x)\left(q_{0}(x)\right.$ and $q_{1}(x)$ in the case of the sG). The proofs that $\left\{\partial_{x}^{l} q(0, t)=g_{l}(t)\right\}_{0}^{n-1}$ and that $\left\{\partial_{x}^{l} q(L, t)=h_{l}(t)\right\}_{0}^{n-1}$ make crucial use of the global relation (1.9). Thus this relation is not only a necessary condition but it is also a sufficient condition for existence. Hence given initial conditions and a subset of $\left\{g_{l}(t), h_{l}(t)\right\}_{0}^{n-1}$ defining a well-posed initial- boundary value problem, the main problem becomes to show that the global relation characterizes the remaining part of $\left\{g_{l}(t), h_{l}(t)\right\}_{0}^{n-1}$.

The analysis of Step 3 is based on the Gelfand-Levitan-Marchenko representation of the eigenfunctions $\Phi(t, k)$ and $\Psi(t, k)$. For example, in the case of the sG equation, it can be shown [9] that $\Phi$ can be expressed in terms of four functions $\left\{M_{j}(t, s), L_{j}(t, s)\right\}_{1}^{2},-t<s<t, t>0$, satisfying a hyperbolic system of four PDEs as well as the Goursat boundary conditions on the characteristics:

$$
\begin{aligned}
L_{1}(t, t) & =-\frac{1}{8}\left(\dot{g}_{0}(t)+g_{1}(t)\right), & L_{2}(t,-t) & =0 \\
M_{1}(t, t) & =-\frac{1}{4} \sin g_{0}(t), & M_{2}(t,-t) & =0 .
\end{aligned}
$$

Similarly, $\Psi$ can be expressed in terms of four functions $\left\{\mathcal{M}_{j}(t, s), \mathcal{L}_{j}(t, s)\right\}_{1}^{2},-t<s<t, t>0$, satisfying

$$
\begin{aligned}
\mathcal{L}_{1}(t, t) & =-\frac{1}{8}\left(\dot{h}_{0}(t)+h_{1}(t)\right), & \mathcal{L}_{2}(t,-t) & =0, \\
\mathcal{M}_{1}(t, t) & =-\frac{1}{4} \sin h_{0}(t), & \mathcal{M}_{2}(t,-t) & =0 .
\end{aligned}
$$

Using the definitions (1.13) and letting $T$ to vary, it can be shown that [9]

$$
\begin{aligned}
& A(t, k)=1+\int_{0}^{t} e^{\frac{i}{2}(k+1 / k) \tau}\left(2 \overline{L_{2}}(t, t-2 \tau)-\frac{i}{k}\left[-\overline{M_{2}}(t, t-2 \tau)+\tan \left(\frac{g_{0}(t)}{2}\right) \overline{M_{1}}(t, t-2 \tau)\right]\right) d \tau, \\
& B(t, k)=-\int_{0}^{t} e^{\frac{i}{2}(k+1 / k) \tau}\left(2 i L_{1}(t, 2 \tau-t)-\frac{1}{k}\left[M_{1}(t, 2 \tau-t)+\tan \left(\frac{g_{0}(t)}{2}\right) M_{2}(t, 2 \tau-t)\right]\right) d \tau .
\end{aligned}
$$

Similar expressions are valid for $\mathcal{A}$ and $\mathcal{B}$, with $L_{j}, M_{j}, g_{0}$ replaced by $\mathcal{L}_{j}, \mathcal{M}_{j}, h_{0}$, respectively. Substituting the expressions for $A, B, \mathcal{A}, \mathcal{B}$ in the global relation (1.9) and letting $k \rightarrow 1 / k$ in the resulting equation, we obtain two relations coupling

$$
g_{0}, h_{0}, L_{j}, M_{j}, \mathcal{L}_{j}, \mathcal{M}_{j}
$$

It is remarkable that these two relations can be explicitly solved for $g_{1}$ and $h_{1}$ in terms of the quantities appearing in (1.19).

Having solved the global relation it is now possible to formulate the final result in terms of the functions $\Phi_{1}, \Phi_{2}, \Psi_{1}, \Psi_{2}$, see equations (4.21) and (4.25).

\section{A Riemann-Hilbert problem formulation under the assump- tion of existence}

Let $\sigma_{j}$ denote the Pauli matrices

$$
\sigma_{1}=\left(\begin{array}{cc}
0 & 1 \\
1 & 0
\end{array}\right), \quad \sigma_{2}=\left(\begin{array}{cc}
0 & -i \\
i & 0
\end{array}\right), \quad \sigma_{3}=\left(\begin{array}{cc}
1 & 0 \\
0 & -1
\end{array}\right)
$$

The sG and the mKdV equations admit the Lax pair formulation (1.7), where $f_{1}, f_{2}, Q$, and $\tilde{Q}$ are given below. 
- sG: $\quad f_{1}(k)=\frac{1}{4}\left(k-\frac{1}{k}\right), f_{2}(k)=\frac{1}{4}\left(k+\frac{1}{k}\right)$,

$$
\begin{aligned}
& Q(x, t, k)=-\frac{i}{4}\left(q_{x}+q_{t}\right) \sigma_{1}-\frac{i \sin q}{4 k} \sigma_{2}+\frac{i}{4 k}(\cos q-1) \sigma_{3}, \\
& \tilde{Q}(x, t, k)=Q(x, t,-k) .
\end{aligned}
$$

- $\operatorname{mKdV~I:~} \quad f_{1}(k)=k, f_{2}(k)=4 k^{3}$,

$$
\begin{aligned}
& Q(x, t, k)=\left(\begin{array}{cc}
0 & q \\
\lambda q & 0
\end{array}\right), \\
& \tilde{Q}(x, t, k)=4 k^{2} Q-2 i k\left(Q^{2}+Q_{x}\right) \sigma_{3}+2 Q^{3}-Q_{x x} .
\end{aligned}
$$

- mKdV II: $\quad f_{1}(k)=-k, f_{2}(k)=4 k^{3}$,

$$
\begin{aligned}
& Q(x, t, k)=\left(\begin{array}{cc}
0 & q \\
\lambda q & 0
\end{array}\right) \\
& \tilde{Q}(x, t, k)=-4 k^{2} Q-2 i k\left(Q^{2}+Q_{x}\right) \sigma_{3}-2 Q^{3}+Q_{x x} .
\end{aligned}
$$

The Lax pair (1.7) can be written as

$$
d\left(e^{i\left(f_{1}(k) x+f_{2}(k) t\right) \hat{\sigma}_{3}} \mu\right)=W
$$

where $W$ is the exact 1 -form defined by

$$
W(x, t, k)=e^{i\left(f_{1}(k) x+f_{2}(k) t\right) \hat{\sigma}_{3}}(Q \mu d x+\tilde{Q} \mu d t) .
$$

Throughout this section we assume that there exists a sufficiently smooth solution $q(x, t), 0<x<L$, $0<t<T$ of the corresponding nonlinear equation.

\subsection{Eigenfunctions}

Define the matrix-valued functions $\mu_{j}$ by

$$
\mu_{j}(x, t, k)=I+\int_{\left(x_{j}, t_{j}\right)}^{(x, t)} e^{-i\left(f_{1}(k) x+f_{2}(k) t\right) \hat{\sigma}_{3}} W(y, \tau, k), \quad j=1,2,3,4,
$$

where $\left(x_{1}, t_{1}\right)=(0, T),\left(x_{2}, t_{2}\right)=(0,0),\left(x_{3}, t_{3}\right)=(L, 0),\left(x_{4}, t_{4}\right)=(L, T)$. If the paths of integration are chosen to be parallel to the $x$ and $t$ axes, then (2.6) yields the following equations:

$$
\begin{aligned}
& \mu_{1}(x, t, k)=I+\int_{0}^{x} e^{-i f_{1}(k)(x-y) \hat{\sigma}_{3}}\left(Q \mu_{1}\right)(y, t, k) d y-e^{-i f_{1}(k) x \hat{\sigma}_{3}} \int_{t}^{T} e^{-i f_{2}(k)(t-\tau) \hat{\sigma}_{3}}\left(\tilde{Q} \mu_{1}\right)(0, \tau, k) d \tau, \\
& \mu_{2}(x, t, k)=I+\int_{0}^{x} e^{-i f_{1}(k)(x-y) \hat{\sigma}_{3}}\left(Q \mu_{2}\right)(y, t, k) d y+e^{-i f_{1}(k) x \hat{\sigma}_{3}} \int_{0}^{t} e^{-i f_{2}(k)(t-\tau) \hat{\sigma}_{3}}\left(\tilde{Q} \mu_{2}\right)(0, \tau, k) d \tau, \\
& \mu_{3}(x, t, k)=I-\int_{x}^{L} e^{-i f_{1}(k)(x-y) \hat{\sigma}_{3}}\left(Q \mu_{3}\right)(y, t, k) d y+e^{-i f_{1}(k)(x-L) \hat{\sigma}_{3}} \int_{0}^{t} e^{-i f_{2}(k)(t-\tau) \hat{\sigma}_{3}}\left(\tilde{Q} \mu_{3}\right)(L, \tau, k) d \tau, \\
& \mu_{4}(x, t, k)=I-\int_{x}^{L} e^{-i f_{1}(k)(x-y) \hat{\sigma}_{3}}\left(Q \mu_{4}\right)(y, t, k) d y-e^{-i f_{1}(k)(x-L) \hat{\sigma}_{3}} \int_{t}^{T} e^{-i f_{2}(k)(t-\tau) \hat{\sigma}_{3}}\left(\tilde{Q} \mu_{4}\right)(L, \tau, k) d \tau .
\end{aligned}
$$




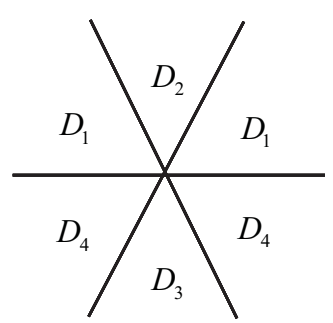

mKdV I

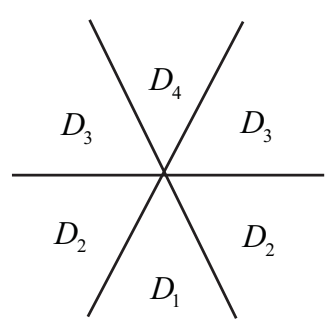

mKdV II

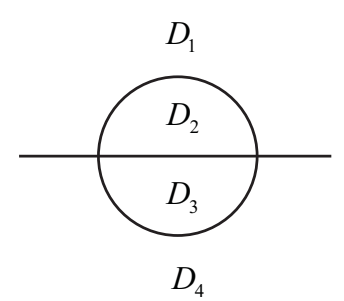

sG

Figure 2: The domains $D_{j}, j=1, \ldots, 4$ for $\mathrm{mKdV} \mathrm{I}, \mathrm{mKdV}$ II, and SG equations

The domains where the exponentials appearing in (2.7) are bounded, are separated by the curves $\left\{k \in \mathbb{C} \mid \operatorname{Im} f_{1}(k) \cdot \operatorname{Im} f_{2}(k)=0\right\}$. Define the domains $D_{j}, j=1,2,3,4$, as follows:

$$
\begin{aligned}
& D_{1}=\left\{k \mid \operatorname{Im} f_{1}(k)>0 \cap \operatorname{Im} f_{2}(k)>0\right\}, \\
& D_{2}=\left\{k \mid \operatorname{Im} f_{1}(k)>0 \cap \operatorname{Im} f_{2}(k)<0\right\}, \\
& D_{3}=\left\{k \mid \operatorname{Im} f_{1}(k)<0 \cap \operatorname{Im} f_{2}(k)>0\right\}, \\
& D_{4}=\left\{k \mid \operatorname{Im} f_{1}(k)<0 \cap \operatorname{Im} f_{2}(k)<0\right\} .
\end{aligned}
$$

Let the columns of a $2 \times 2$ matrix $\mu$ be denoted as $\left(\mu^{(1)} \mu^{(2)}\right)$. Then the columns of $\mu_{j}$ are analytic and bounded in the following domains in the complex $k$-plane (which are determined by the domains of boundedness of the exponentials involved in the relative integral equations):

$$
\begin{array}{lll}
\mu_{1}^{(1)}, \mu_{3}^{(2)} & : & D_{2}, \\
\mu_{1}^{(2)}, \mu_{3}^{(1)} & : & D_{3}, \\
\mu_{2}^{(1)}, \mu_{4}^{(2)} & : & D_{1}, \\
\mu_{2}^{(2)}, \mu_{4}^{(1)} & : & D_{4} .
\end{array}
$$

Thus, in each $D_{j}$ there exists a $2 \times 2$ matrix-valued eigenfunction, which is analytic and bounded. For example, in $D_{1}$ the matrix is $\left(\mu_{2}^{(1)} \mu_{4}^{(2)}\right)$, etc.

For particular values of $x$ or $t$, the domains of boundedness of the eigenfunctions are larger than indicated above. In particular, for $t=0$, the domain of boundedness of $\mu_{2}$ are the following:

$$
\begin{array}{lll}
\mu_{2}^{(1)}(x, 0, k), \mu_{3}^{(2)}(x, 0, k) & : & \left\{\operatorname{Im} f_{1}(k)>0\right\}=D_{1} \cup D_{2}, \\
\mu_{2}^{(2)}(x, 0, k), \mu_{3}^{(1)}(x, 0, k) & : & \left\{\operatorname{Im} f_{1}(k)<0\right\}=D_{3} \cup D_{4} .
\end{array}
$$

For $x=0$, the domain of boundedness of $\mu_{1}$ and $\mu_{2}$ are the following:

$$
\begin{array}{lll}
\mu_{1}^{(1)}(0, t, k) \text { and } \mu_{2}^{(2)}(0, t, k) & : & \left\{\operatorname{Im} f_{2}(k)<0\right\}=D_{2} \cup D_{4}, \\
\mu_{1}^{(2)}(0, t, k) \text { and } \mu_{2}^{(1)}(0, t, k): & : & \left\{\operatorname{Im} f_{2}(k)>0\right\}=D_{1} \cup D_{3} .
\end{array}
$$

For $x=L$, the domain of boundedness of $\mu_{3}$ and $\mu_{4}$ are the following:

$$
\begin{aligned}
& \mu_{3}^{(2)}(L, t, k) \text { and } \mu_{4}^{(1)}(L, t, k) \quad: \quad\left\{\operatorname{Im} f_{2}(k)<0\right\}=D_{2} \cup D_{4}, \\
& \mu_{3}^{(1)}(L, t, k) \text { and } \mu_{4}^{(2)}(L, t, k):\left\{\operatorname{Im} f_{2}(k)>0\right\}=D_{1} \cup D_{3} \text {. }
\end{aligned}
$$

\subsection{Other properties of eigenfunctions}

The matrices $Q$ and $\tilde{Q}$ have certain symmetry properties, which, in turn imply the following symmetries for $\mu$ :

$$
(\mu(x, t, k))_{11}=\overline{(\mu(x, t, \bar{k}))_{22}}, \quad(\mu(x, t, k))_{12}=\rho \overline{(\mu(x, t, \bar{k}))_{21}}
$$


and

$$
\mu(x, t,-k)=\overline{\mu(x, t, \bar{k})}
$$

In the domains where $\mu$ is bounded,

$$
\mu(x, t, k)=I+O\left(\frac{1}{k}\right), \quad|k| \rightarrow \infty .
$$

The fact that $Q$ and $\tilde{Q}$ are traceless together with (2.11) imply

$$
\operatorname{det} \mu(x, t, k)=1 \text {. }
$$

\subsection{Spectral functions}

Since the eigenfunctions $\mu_{j}$ are solutions of the system of differential equations (1.7), they are simply related as follows:

$$
\begin{aligned}
& \mu_{3}(x, t, k)=\mu_{2}(x, t, k) e^{-i\left(f_{1}(k) x+f_{2}(k) t\right) \hat{\sigma}_{3}} s(k), \\
& \mu_{1}(x, t, k)=\mu_{2}(x, t, k) e^{-i\left(f_{1}(k) x+f_{2}(k) t\right) \hat{\sigma}_{3}} S(k), \\
& \mu_{4}(x, t, k)=\mu_{3}(x, t, k) e^{-i\left(f_{1}(k)(x-L)+f_{2}(k) t\right) \hat{\sigma}_{3}} S_{L}(k) .
\end{aligned}
$$

Evaluating (2.13a) at $x=t=0$, we find

$$
s(k)=\mu_{3}(0,0, k) .
$$

Evaluating (2.13b) at $x=t=0$, we find

$$
S(k)=\mu_{1}(0,0, k)
$$

evaluating (2.13b) at $x=0, t=T$, we find $S(k)=\left(e^{i f_{2}(k) T \hat{\sigma}_{3}} \mu_{2}(0, T, k)\right)^{-1}$.

Evaluating (2.13c) at $x=L, t=0$, we find

$$
S_{L}(k)=\mu_{4}(L, 0, k)
$$

evaluating (2.13c) at $x=L, t=T$, we find $S_{L}(k)=\left(e^{i f_{2}(k) T \hat{\sigma}_{3}} \mu_{3}(L, T, k)\right)^{-1}$.

Equations (2.13a), (2.13b) imply

$$
\mu_{4}(x, t, k)=\mu_{2}(x, t, k) e^{-i\left(f_{1}(k) x+f_{2}(k) t\right) \hat{\sigma}_{3}} s(k) e^{i f_{1}(k) L \hat{\sigma}_{3}} S_{L}(k) .
$$

The definitions of $s(k), S(k)$, and $S_{L}(k)$ imply the following results:

$$
s(k)=I-\int_{0}^{L} e^{i f_{1}(k) y \hat{\sigma}_{3}}\left(Q \mu_{3}\right)(y, 0, k) d y,
$$

where $\mu_{3}(x, 0, k), 0<x<L$, is the solution of the integral equation

$$
\mu_{3}(x, 0, k)=I-\int_{x}^{L} e^{-i f_{1}(k)(x-y) \hat{\sigma}_{3}}\left(Q \mu_{3}\right)(y, 0, k) d y .
$$

Similarly,

$$
\begin{aligned}
S(k) & =\left(I+\int_{0}^{T} e^{i f_{2}(k) \tau \hat{\sigma}_{3}}\left(\tilde{Q} \mu_{2}\right)(0, \tau, k) d \tau\right)^{-1}, \\
S_{L}(k) & =\left(I+\int_{0}^{T} e^{i f_{2}(k) \tau \hat{\sigma}_{3}}\left(\tilde{Q} \mu_{3}\right)(L, \tau, k) d \tau\right)^{-1},
\end{aligned}
$$


where $\mu_{2}(0, t, k)$ and $\mu_{3}(L, t, k), 0<t<T$ are the solutions of the integral equations

$$
\begin{aligned}
& \mu_{2}(0, t, k)=I+\int_{0}^{t} e^{-i f_{2}(k)(t-\tau) \hat{\sigma}_{3}}\left(\tilde{Q} \mu_{2}\right)(0, \tau, k) d \tau, \\
& \mu_{3}(L, t, k)=I+\int_{0}^{t} e^{-i f_{2}(k)(t-\tau) \hat{\sigma}_{3}}\left(\tilde{Q} \mu_{3}\right)(L, \tau, k) d \tau,
\end{aligned}
$$

respectively. Note that $Q(x, 0)$ is determined by $q(x, 0)\left(q(x, 0)\right.$ and $q_{t}(x, 0)$ for the $\left.\mathrm{sG}\right), \tilde{Q}(0, t, k)$ is determined by $\left\{\partial_{x}^{l} q(0, t)\right\}_{0}^{n-1}$, and $\tilde{Q}(L, t, k)$ is determined by $\left\{\partial_{x}^{l} q(L, t)\right\}_{0}^{n-1}$.

The symmetry properties (1.12) of the spectral functions follow from the symmetry properties (2.9) of the associated eigenfunctions.

Equations (2.18) - (2.23) and the determinant condition (2.12) imply the following properties of the spectral functions:

$\underline{a(k), b(k)}$

- For the mKdV I and II, $a(k)$ and $b(k)$ are entire functions; for the sG, $a(k)$ and $b(k)$ are analytic in the punctured plane $\mathbb{C} \backslash\{0\}$.

- $a(k), b(k), \overline{a(\bar{k})} e^{2 i f_{1}(k) L}, \overline{b(\bar{k})} e^{2 i f_{1}(k) L}$ are bounded in $\bar{D}_{1} \cup \bar{D}_{2}$ (here $\bar{D}$ denotes the closure of a domain $D)$.

$$
a(k)=1+O\left(\frac{1+e^{2 i f_{1}(k) L}}{k}\right), \quad b(k)=O\left(\frac{1+e^{2 i f_{1}(k) L}}{k}\right), \quad|k| \rightarrow \infty .
$$

- $a(k) \overline{a(\bar{k})}-\rho b(k) \overline{b(\bar{k})}=1, \quad k \in \mathbb{C}$.

- $a(-k)=\overline{a(\bar{k})}, \quad b(-k)=\overline{b(\bar{k})}$.

$\underline{A(k), B(k)}$

- For the mKdV I and II, $A(k)$ and $B(k)$ are entire functions; for the sG, $A(k)$ and $B(k)$ are analytic in the punctured plane $\mathbb{C} \backslash\{0\}$.

- $A(k)$ and $B(k)$ are bounded in $\bar{D}_{1} \cup \bar{D}_{3}$.

-

$$
A(k)=1+O\left(\frac{1+e^{2 i f_{2}(k) T}}{k}\right), \quad B(k)=O\left(\frac{1+e^{2 i f_{2}(k) T}}{k}\right), \quad k \rightarrow \infty .
$$

- $A(k) \overline{A(\bar{k})}-\rho B(k) \overline{B(\bar{k})}=1, \quad k \in \mathbb{C}$.

- $A(-k)=\overline{A(\bar{k})}, \quad B(-k)=\overline{B(\bar{k})}$.

$\mathcal{A}(k), \mathcal{B}(k)$

Same as $A(k), B(k)$.

We note that $q(x, 0)\left(q(x, 0)\right.$ and $q_{t}(x, 0)$ for the sG equation) can be expressed in terms of $\{a(k), b(k)\}$ through the solution of a Riemann-Hilbert problem [6]. This problem is a singular RH problem: its solution can have poles (at possible zeros of $a$ and $\bar{a}$ ), therefore, residue relations have to be added to its formulations. Here we present an alternative construction of the $\mathrm{RH}$ problem, which is regular relative to an augmented contour containing additional parts $\left\{k \in \mathbb{C}:|k|=R_{j}\right\}$ surrounding the poles of $f_{1}(k)$ and $f_{2}(k)$ (see [13]). In such a formulation, we need not any hypothesis on zeros of $a(k)$. 


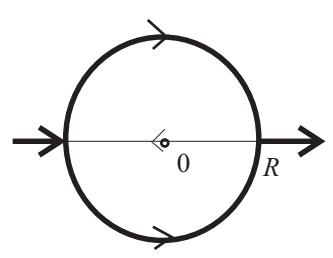

mKdV I

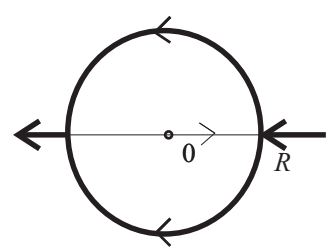

$\mathrm{mKdV}$ II

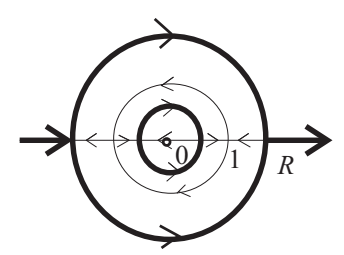

sG

Figure 3: The oriented contours $\Sigma^{(x)}$ for the mKdV I, mKdV II, and SG equations (jump across the thin part of the contours is trivial)

Define the domain $D_{0}$ as follows:

$$
D_{0}=\left\{\begin{array}{ll}
|k|<R & \text { for MKdV I and II } \\
\varepsilon<|k|<R & \text { for } \mathrm{sG}
\end{array},\right.
$$

where $\varepsilon$ and $R$ are chosen such that all the zeros of $a(k)$ from $D_{1} \cup D_{2}$ are in $D_{0}$.

Define a sectionally holomorphic, matrix-valued function $M^{(x)}(x, k)$ :

$$
M^{(x)}(x, k)=\left\{\begin{array}{cl}
\left(\mu_{3}^{(1)}(x, 0, k) \frac{\mu_{2}^{(2)}(x, 0, k)}{\overline{a(\bar{k})}}\right) & k \in\left(D_{3} \cup D_{4}\right) \backslash \bar{D}_{0} \\
\left(\frac{\mu_{2}^{(1)}(x, 0, k)}{a(k)} \mu_{3}^{(2)}(x, 0, k)\right) & k \in\left(D_{1} \cup D_{2}\right) \backslash \bar{D}_{0} \\
\mu_{2}(x, 0, k) & k \in D_{0} .
\end{array}\right.
$$

Similarly to $\mu_{3}(x, 0, k), \mu_{2}(x, 0, k)$ in $(2.27)$ is determined by $(2.7 \mathrm{~b})$ for $t=0$. Then the limits $M_{ \pm}^{(x)}(x, \zeta)$ of $M^{(x)}(x, k)$ as $k$ approaches the oriented contour $\Sigma^{(x)}$ in the complex $k$-plane from the corresponding side (see Figure 3 ) are related by the jump matrix $J^{(x)}(x, \zeta)$ :

$$
M_{-}^{(x)}(x, \zeta)=M_{+}^{(x)}(x, \zeta) J^{(x)}(x, \zeta), \quad \zeta \in \Sigma^{(x)},
$$

where

$$
J^{(x)}(x, k)=\left\{\begin{array}{cc}
\left(\begin{array}{cc}
1 & -\frac{b(k)}{\bar{a}(k)} e^{-2 i f_{1}(k) x} \\
\rho \frac{\bar{b}(k)}{a(k)} e^{2 i f_{1}(k) x} & 1-\rho\left|\frac{b(k)}{\bar{a}(k)}\right|^{2}
\end{array}\right) & k \in \Gamma_{1} \\
I & k \in \partial\left\{D_{1} \cup D_{2}\right\} \cap \bar{D}_{0} \\
\left(\begin{array}{cc}
\frac{a(\bar{k})}{\rho(\bar{k})} e^{-2 i f_{1}(k) x} & \frac{1}{\overline{a(\bar{k})}}
\end{array}\right) & k \in \partial\left\{D_{3} \cup D_{4} \backslash \bar{D}_{0}\right\} \backslash \bar{\Gamma}_{1} \\
\left(\begin{array}{cc}
a(k) & -b(k) e^{-2 i f_{1}(k) x} \\
0 & \frac{1}{a(k)}
\end{array}\right) & k \in \partial\left\{D_{1} \cup D_{2} \backslash \bar{D}_{0}\right\} \backslash \bar{\Gamma}_{1}
\end{array}\right.
$$

with $\Gamma_{1}=\partial\left\{D_{1} \cup D_{2}\right\} \backslash \bar{D}_{0}$; here $\partial\{D\}$ denotes the boundary of a domain $D$. 


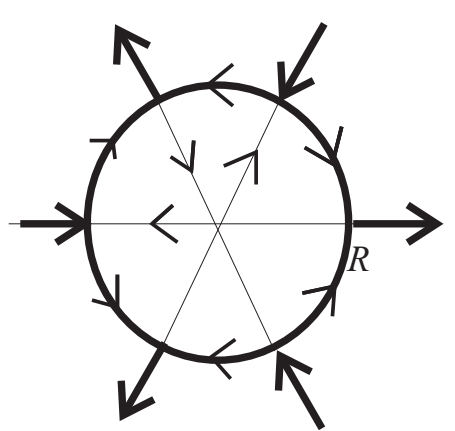

mKdV I,II

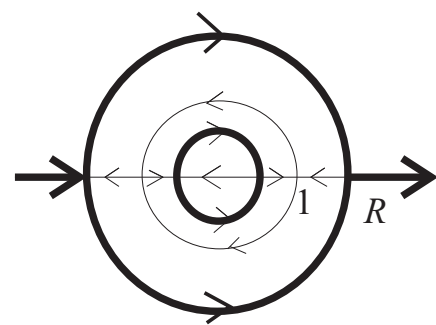

sG

Figure 4: The oriented contours $\Sigma$ for the $\mathrm{mKdV}$ and sG equations (the jump across the thin part of the contours is trivial)

Define a sectionally holomorphic, matrix-valued function $M^{(t, 0)}(t, k)$ :

$$
M^{(t, 0)}(t, k)=\left\{\begin{array}{cl}
\left(\mu_{1}^{(1)}(0, t, k) \frac{\mu_{2}^{(2)}(0, t, k)}{\overline{A(\bar{k})}}\right) & k \in\left(D_{2} \cup D_{4}\right) \backslash \bar{D}_{0} \\
\left(\frac{\mu_{2}^{(1)}(0, t, k)}{A(k)} \mu_{1}^{(2)}(0, t, k)\right) & k \in\left(D_{1} \cup D_{3}\right) \backslash \bar{D}_{0} \\
\mu_{2}(0, t, k) & k \in D_{0},
\end{array}\right.
$$

where $D_{0}$ is of the form (2.26) containing all the zeros of $A(k)$ in $D_{1} \cup D_{3}$. Similarly to $\mu_{2}(0, t, k)$, $\mu_{1}(0, t, k)$ in (2.30) is determined by (2.7a) for $x=0$. Then the limits $M_{ \pm}^{(t, 0)}(t, \zeta)$ of $M^{(t, 0)}(t, k)$ as $k$ approaches the contour $\Sigma$ in the complex $k$-plane, see Figure 4 , are related by the jump matrix $J^{(t, 0)}(t, \zeta)$ :

$$
M_{-}^{(t, 0)}(t, \zeta)=M_{+}^{(t, 0)}(t, \zeta) J^{(t, 0)}(t, \zeta), \quad \zeta \in \Sigma,
$$

where

$$
J^{(t, 0)}(t, k)=\left\{\begin{array}{cc}
\left(\begin{array}{cc}
1 & -\frac{B(k)}{\overline{A(\bar{k})}} e^{-2 i f_{2}(k) t} \\
\frac{\rho \overline{B(\bar{k})}}{A(k)} e^{2 i f_{2}(k) t} & \frac{1}{A(k) \overline{A(\bar{k})}}
\end{array}\right) & k \in \Gamma_{2} \\
I & k \in \partial\left\{D_{1} \cup D_{3}\right\} \cap \bar{D}_{0} \\
\left(\begin{array}{cc}
A(k) & -B(k) e^{-2 i f_{2}(k) t} \\
0 & \frac{1}{A(k)}
\end{array}\right) & k \in \partial\left\{\left(D_{1} \cup D_{3}\right) \backslash \bar{D}_{0}\right\} \backslash \bar{\Gamma}_{2} \\
\left(\begin{array}{cc}
\overline{A(\bar{k})} & 0 \\
\lambda \overline{B(\bar{k})} e^{2 i f_{2}(k) t} & \frac{1}{\overline{A(\bar{k})}}
\end{array}\right) & k \in \partial\left\{\left(D_{2} \cup D_{4}\right) \backslash \bar{D}_{0}\right\} \backslash \bar{\Gamma}_{2}
\end{array}\right.
$$

with $\Gamma_{2}=\partial\left\{D_{1} \cup D_{3}\right\} \backslash \bar{D}_{0}$. 
Similarly, define a sectionally holomorphic, matrix-valued function $M^{(t, L)}(t, k)$ :

$$
M^{(t, L)}(t, k)=\left\{\begin{array}{cl}
\left(\mu_{4}^{(1)}(L, t, k) \frac{\mu_{3}^{(2)}(L, t, k)}{\overline{\mathcal{A}(\bar{k})})}\right. & k \in\left(D_{2} \cup D_{4}\right) \backslash \bar{D}_{0} \\
\left(\frac{\mu_{3}^{(1)}(L, t, k)}{\mathcal{A}(k)} \mu_{4}^{(2)}(L, t, k)\right) & k \in\left(D_{1} \cup D_{3}\right) \backslash \bar{D}_{0} \\
\mu_{3}(L, t, k) & k \in D_{0},
\end{array}\right.
$$

where $D_{0}$ is of the form (2.26) containing all the zeros of $\mathcal{A}(k)$ in $D_{1} \cup D_{3}$. Similarly to $\mu_{3}(L, t, k)$, $\mu_{4}(L, t, k)$ in $(2.33)$ is determined by $(2.7 \mathrm{~d})$ for $x=L$. Then the limits $M_{ \pm}^{(t, L)}(t, \zeta)$ of $M^{(t, L)}(t, k)$ as $k$ approaches the contour $\Sigma$ are related by the jump matrix $J^{(t, L)}(t, \zeta)$ :

$$
M_{-}^{(t, L)}(t, \zeta)=M_{+}^{(t, L)}(t, \zeta) J^{(t, L)}(t, \zeta), \quad \zeta \in \Sigma,
$$

where $J^{(t, L)}(t, \zeta)$ is constructed similarly to $(2.32)$ with $A(k), B(k)$ replaced by $\mathcal{A}(k), \mathcal{B}(k)$.

\subsection{Global relation}

Evaluating equation (2.17) at $x=0, t=T$, and writing $\mu_{2}(0, T, k)$ in terms of $S(k)$ we find

$$
\mu_{4}(0, T, k)=e^{-i f_{2}(k) T \hat{\sigma}_{3}} S^{-1}(k) s(k) e^{i f_{1}(k) L \hat{\sigma}_{3}} S_{L}(k) .
$$

Multiplying this equation by $\exp \left\{i f_{2}(k) T \hat{\sigma}_{3}\right\}$ and using the definition of $\mu_{4}(x, T, k)$ we find

$$
-I+S^{-1}(k) s(k)\left[e^{i f_{1}(k) L \hat{\sigma}_{3}} S_{L}(k)\right]+e^{i f_{2}(k) T \hat{\sigma}_{3}} \int_{0}^{L} e^{i f_{1}(k) y \hat{\sigma}_{3}}\left(Q \mu_{4}\right)(y, T, k) d y=0 .
$$

The (12) element of $(2.35)$ is $(k \in \mathbb{C})$

$$
e^{2 i f_{1}(k) L}(\overline{a(\bar{k})} A(k)-\lambda \overline{b(\bar{k})} B(k)) \mathcal{B}(k)-(a(k) B(k)-b(k) A(k)) \mathcal{A}(k)=e^{2 i f_{2}(k) T} c(k),
$$

where

$$
c(k)=c(k, T)=-\int_{0}^{L} e^{2 i f_{1}(k) y}\left(Q \mu_{4}\right)_{12}(y, T, k) d y=\left(\mu_{4}\right)_{12}(0, T, k)
$$

is of $O\left(\left(1+e^{2 i f_{1}(k) L}\right) / k\right)$ as $k \rightarrow \infty$.

Equation (2.36) expresses, in the spectral terms, the relation between the initial and boundary values of the solution of the given nonlinear equation.

\subsection{The jump conditions for the eigenfunctions}

Relating the vector solutions of (1.7) in the respective domains by using (2.13) and the definitions of the spectral functions (2.14)-(2.16), we find

$$
M_{-}(x, t, k)=M_{+}(x, t, k) J(x, t, k), \quad k \in \Sigma,
$$


where $M_{ \pm}(x, t, k)$ are the limit values (as $k$ approaches $\Sigma$ from $\Omega_{ \pm}$) of a sectionally holomorphic function $M(x, t, k)$ defined as follows:

$$
M= \begin{cases}\left(\mu_{3}^{(1)} \frac{\mu_{1}^{(2)}}{\overline{d(\bar{k})}}\right), & k \in D_{3} \backslash \bar{D}_{0} \\ \left(\frac{\mu_{4}^{(1)} \overline{a(\bar{k})}}{\overline{d_{1}(\bar{k})}} \frac{\mu_{2}^{(2)}}{\overline{a(\bar{k})}}\right), & k \in D_{4} \backslash \bar{D}_{0} \\ \left(\frac{\mu_{1}^{(1)}}{d(k)} \mu_{3}^{(2)}\right), & k \in D_{2} \backslash \bar{D}_{0} \\ \left(\frac{\mu_{2}^{(1)}}{a(k)} \frac{\mu_{4}^{(2)} a(k)}{d_{1}(k)}\right), & k \in D_{1} \backslash \bar{D}_{0} \\ \mu_{2}, & k \in D_{0}\end{cases}
$$

where

$$
\begin{aligned}
d(k) & =a(k) \overline{A(\bar{k})}-\rho b(k) \overline{B(\bar{k})}, \quad k \in D_{2} \cup D_{4}, \\
d_{1}(k) & =a(k) A_{1}(k)+\rho e^{2 i f_{1}(k) L} \overline{b(\bar{k})} B_{1}(k), \quad k \in D_{1} \cup D_{3} .
\end{aligned}
$$

The jump matrix $J(x, t, k)$ has explicit $(x, t)$ dependence:

$$
J(x, t, k)=e^{\left(-i f_{1}(k) x-i f_{2}(k) t\right) \hat{\sigma}_{3}} J_{0}(k),
$$

where $J_{0}(k)$ is constructed from the elements of the spectral functions:

- For $k \in \Sigma, \operatorname{Im} f_{1}(k) \leq 0$ we have

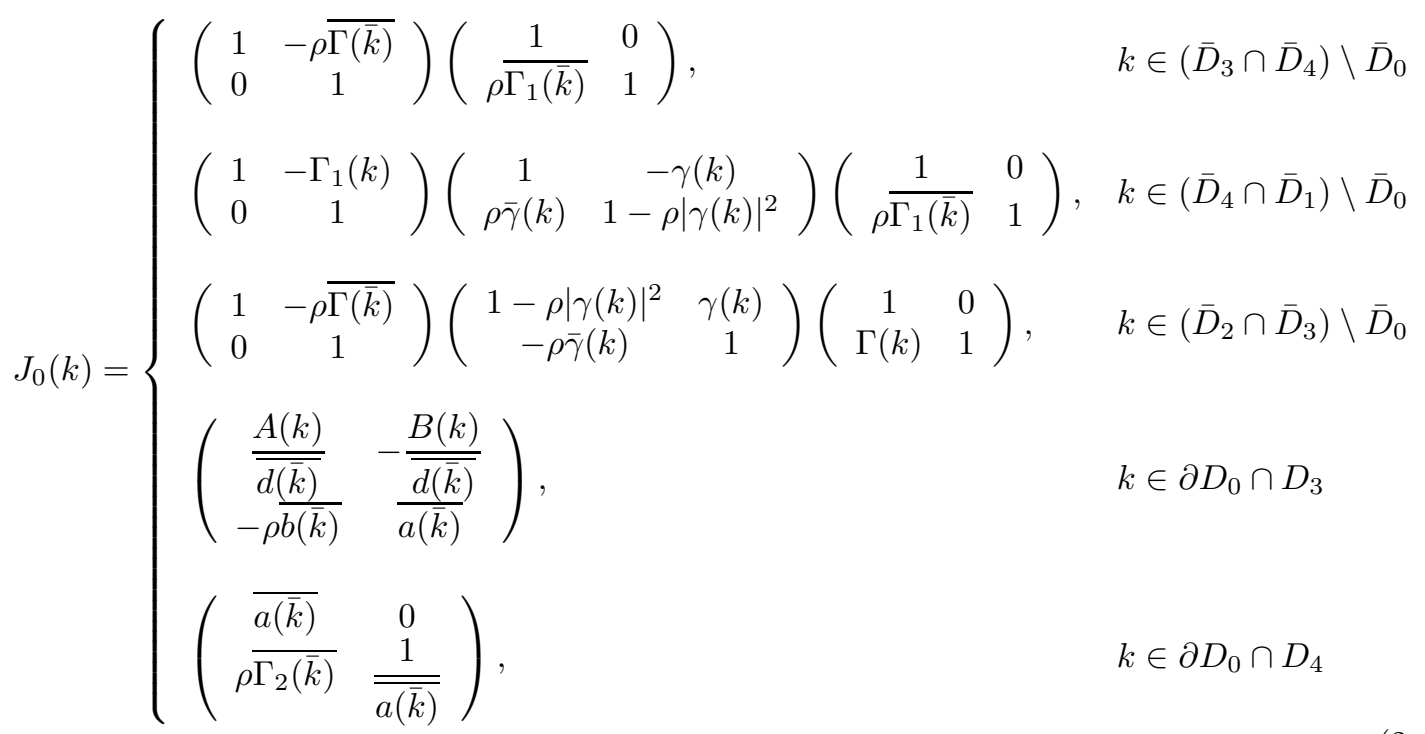

- For $k \in \Sigma, \operatorname{Im} f_{1}(k)>0$ we have

$$
J_{0}(k)=\left(\begin{array}{cc}
-1 & 0 \\
0 & \rho
\end{array}\right) J_{0}^{*}(\bar{k})\left(\begin{array}{cc}
-1 & 0 \\
0 & \rho
\end{array}\right)
$$

- For $k \in \Sigma, k \in D_{0}$ we have $J_{0}(k)=I$. 
Here

$$
\begin{aligned}
& \gamma(k)=\frac{b(k)}{\bar{a}(k)}, \quad \Gamma(k)=\frac{\lambda \overline{B(\bar{k})}}{a(k) d(k)}, \\
& \Gamma_{1}(k)=\frac{e^{2 i f_{1}(k) L} a(k) \mathcal{B}(k)}{d_{1}(k)}, \quad \Gamma_{2}(k)=\frac{\Gamma_{1}(k)}{a(k)}+b(k),
\end{aligned}
$$

and the parameters $\varepsilon$ and $R$ determining the domain $D_{0}$ are chosen such that all zeros of $a(k), d(k)$, and $d_{1}(k)$ from $\operatorname{Im} f_{1}(k) \geq 0$ are in $D_{0}$.

\section{Existence under assumption that the global relation is valid}

The analysis of section 2 motivates the following definitions and results.

Definition 1. Denote by $Q^{(x)}(x, k)$ the matrix $Q(x, 0, k)$, in which $q(x, 0)\left(q(x, 0)\right.$ and $q_{t}(x, 0)$ for the $s G)$ are replaced by $q_{0}(x)\left(q_{0}(x)\right.$ and $q_{1}(x)$, respectively).

Given a smooth function $q_{0}(x)$ (two smooth functions, $q_{0}(x)$ and $q_{1}(x)$, for the $s G$ ), define the vector $\varphi(x, k)=\left(\varphi_{1}, \varphi_{2}\right)^{T}$ as the unique solution of

$$
\begin{aligned}
& \varphi_{1_{x}}+2 i f_{1}(k) \varphi_{1}=Q_{11}^{(x)} \varphi_{1}+Q_{12}^{(x)} \varphi_{2}, \\
& \varphi_{2 x}=Q_{21}^{(x)} \varphi_{1}+Q_{22}^{(x)} \varphi_{2}, \quad 0<x<L \quad k \in \mathbb{C} \\
& \varphi(L, k)=(0,1)^{T} .
\end{aligned}
$$

Given $\varphi(x, k)$ define the functions $a(k)$ and $b(k)$ by

$$
a(k)=\varphi_{2}(0, k), \quad b(k)=\varphi_{1}(0, k), \quad k \in \mathbb{C} .
$$

Then $a(k)$ and $b(k)$ have the properties (2.24). Definition 1 gives rise to the map

$$
\mathbb{S}:\left\{q_{0}(x)\right\} \mapsto\{a(k), b(k)\}
$$

$\left(\left\{q_{0}(x), q_{1}(x)\right\} \mapsto\{a(k), b(k)\}\right.$ in the case of the $\left.\mathrm{sG}\right)$. The inverse of this map,

$$
\mathbb{Q}:\{a(k), b(k)\} \mapsto\left\{q_{0}(x)\right\}
$$

$\left(\{a(k), b(k)\} \mapsto\left\{q_{0}(x), q_{1}(x)\right\}\right.$ in the case of the $\left.\mathrm{sG}\right)$ can be defined as follows:

$$
\begin{aligned}
m K d V I: \quad q_{0}(x) & =2 i \lim _{k \rightarrow \infty}\left(k M^{(x)}(x, k)\right)_{12} ; \\
m K d V I I: \quad q_{0}(x) & =-2 i \lim _{k \rightarrow \infty}\left(k M^{(x)}(x, k)\right)_{12} ; \\
s G: \quad \cos q_{0}(x) & =1+2 \lim _{k \rightarrow \infty}\left\{\left(k M^{(x)}(x, k)\right)_{12}^{2}+2 i \partial_{x}\left(k M^{(x)}(x, k)\right)_{22}\right\}, \\
q_{1}(x) & =-\frac{d q_{0}}{d x}(x)-2 \lim _{k \rightarrow \infty}\left(k M^{(x)}(x, k)\right)_{12},
\end{aligned}
$$

where $M^{(x)}(x, k)$ is the solution of the following $\mathrm{RH}$ problem:

- $M^{(x)}(x, k)$ is a sectionally holomorphic function relative to the contour $\Sigma^{(x)}$.

- the limits $M_{ \pm}^{(x)}(x, \zeta)$ of $M^{(x)}(x, k)$ as $k$ approaches $\Sigma^{(x)}$ are related by (2.28), where the jump matrix $J^{(x)}(x, \zeta)$ is constructed from $a(k)$ and $b(k)$ following (2.29).

- $M^{(x)}(x, k)=I+O\left(\frac{1}{k}\right), \quad k \rightarrow \infty$. 
Definition 2. Denote by $Q^{(t, 0)}(t, k)$ the matrix $\tilde{Q}(0, t, k)$, in which $\partial q_{x}^{l}(0, t)$ are replaced by $g_{l}(t), 0 \leq$ $l \leq n-1$.

Given smooth functions $g_{l}(t), 0 \leq l \leq n-1$, define the vector $\Phi(t, k)=\left(\Phi_{1}, \Phi_{2}\right)^{T}$ as the unique solution of

$$
\begin{aligned}
& \Phi_{1 t}+2 i f_{2}(k) \Phi_{1}=Q_{11}^{(t, 0)} \Phi_{1}+Q_{12}^{(t, 0)} \Phi_{2}, \\
& \Phi_{2 t}=Q_{21}^{(t, 0)} \Phi_{1}+Q_{22}^{(t, 0)} \Phi_{2}, \quad 0<t<T \quad k \in \mathbb{C} \\
& \Phi(0, k)=(0,1)^{T} .
\end{aligned}
$$

Given $\Phi(t, k)$ define the functions $A(k)$ and $B(k)$ by

$$
A(k)=\overline{\Phi_{2}(T, \bar{k})}, \quad B(k)=-\Phi_{1}(T, k) e^{2 i f_{2}(k) T}, \quad k \in \mathbb{C} .
$$

Then $A(k)$ and $B(k)$ have the properties (2.25). Definition 2 gives rise to the map

$$
\mathbb{S}^{(0)}:\left\{g_{l}(t), 0 \leq l \leq n-1\right\} \mapsto\{A(k), B(k)\} .
$$

The inverse of this map,

$$
\mathbb{Q}^{(0)}:\{A(k), B(k)\} \mapsto\left\{g_{l}(t), 0 \leq l \leq n-1\right\}
$$

can be defined as follows:

$$
\begin{aligned}
m K d V I: \quad g_{0}(t) & =2 i \lim _{k \rightarrow \infty} k\left(M^{(t, 0)}(t, k)\right)_{12}, \\
g_{1}(t) & =\lim _{k \rightarrow \infty}\left\{4 k^{2}\left(M^{(t, 0)}(t, k)\right)_{12}+2 i g_{0}(t) k\left(M^{(t, 0)}(t, k)\right)_{22}\right\}, \\
g_{2}(t) & =\lim _{k \rightarrow \infty}\left\{\rho g_{0}^{3}(t)-8 i k^{3}\left(M^{(t, 0)}(t, k)\right)_{12}+4 g_{0}(t) k^{2}\left(M^{(t, 0)}(t, k)\right)_{22}\right. \\
& \left.+2 i g_{1}(t) k\left(M^{(t, 0)}(t, k)\right)_{22}\right\} ; \\
m K d V I I: \quad g_{0}(t) & =-2 i \lim _{k \rightarrow \infty} k\left(M^{(t, 0)}(t, k)\right)_{12}, \\
g_{1}(t) & =\lim _{k \rightarrow \infty}\left\{4 k^{2}\left(M^{(t, 0)}(t, k)\right)_{12}-2 i g_{0}(t) k\left(M^{(t, 0)}(t, k)\right)_{22}\right\}, \\
g_{2}(t) & =\lim _{k \rightarrow \infty}\left\{\rho g_{0}^{3}(t)+8 i k^{3}\left(M^{(t, 0)}(t, k)\right)_{12}+4 g_{0}(t) k^{2}\left(M^{(t, 0)}(t, k)\right)_{22}\right. \\
& \left.-2 i g_{1}(t) k\left(M^{(t, 0)}(t, k)\right)_{22}\right\} ; \\
s G: \quad \cos g_{0}(t) & =1-2 \lim _{k \rightarrow \infty}\left\{\left(k M^{(t, 0)}(t, k)\right)_{12}^{2}+2 i \frac{d}{d t} \lim _{k \rightarrow \infty}\left(k M^{(t, 0)}(t, k)\right)_{22}\right\}, \\
g_{1}(t) & =-\frac{d}{d t} g_{0}(t)-2 \lim _{k \rightarrow \infty}\left(k M^{(t, 0)}(t, k)\right)_{12},
\end{aligned}
$$

where $M^{(t, 0)}(t, k)$ is the solution of the following $\mathrm{RH}$ problem:

- $M^{(t, 0)}(t, k)$ is a sectionally holomorphic function relative to the contour $\Sigma$.

- the limits $M_{ \pm}^{(t, 0)}(t, \zeta)$ of $M^{(t, 0)}(t, k)$ as $k$ approaches $\Sigma$ from $\Omega_{ \pm}$are related by (2.31), where the jump matrix $J^{(t, 0)}(t, \zeta)$ is constructed from $A(k)$ and $B(k)$ following (2.32).

- $M^{(t, 0)}(t, k)=I+O\left(\frac{1}{k}\right), \quad k \rightarrow \infty$.

Definition 3. Denote by $Q^{(t, L)}(t, k)$ the matrix $\tilde{Q}(L, t, k)$, in which $\partial q_{x}^{l}(L, t)$ are replaced by $h_{l}(t)$, $0 \leq l \leq n-1$.

Given smooth functions $h_{l}(t), 0 \leq l \leq n-1$, define the vector $\Psi(t, k)=\left(\Psi_{1}, \Psi_{2}\right)^{T}$ by equations similar to (3.5) with $Q^{(t, 0)}$ replaced by $Q^{(t, L)}$.

Given $\Psi(t, k)$ define the functions $\mathcal{A}(k)$ and $\mathcal{B}(k)$ by

$$
\mathcal{A}(k)=\overline{\Psi_{2}(T, \bar{k})}, \quad \mathcal{B}(k)=-\Psi_{1}(T, k) e^{2 i f_{2}(k) T}, \quad k \in \mathbb{C} .
$$




$$
\mathbb{S}^{(L)}:\left\{h_{l}(t), 0 \leq l \leq n-1\right\} \mapsto\{\mathcal{A}(k), \mathcal{B}(k)\}
$$

and

$$
\mathbb{Q}^{(L)}:\{\mathcal{A}(k), \mathcal{B}(k)\} \mapsto\left\{h_{l}(t), 0 \leq l \leq n-1\right\}
$$

are defined exactly as above, the notations $M^{(t, L)}(t, k)$ and $J^{(t, L)}(t, \zeta)$ being used instead of $M^{(t, 0)}(t, k)$ and $J^{(t, 0)}(t, \zeta)$, respectively.

Definition 4. Given the smooth function $q_{0}(x)\left(q_{0}(x)\right.$ and $q_{1}(x)$ for the $s G$ equation) define $a(k), b(k)$ according to definition 1. Suppose that there exist smooth functions $\left\{g_{l}(t)\right\}_{0}^{n-1}$ and $\left\{h_{l}(t)\right\}_{0}^{n-1}$ such that:

- The associated $A(k), B(k), \mathcal{A}(k), \mathcal{B}(k)$, defined according to definitions 2 and 3, satisfy the relation

$$
e^{2 i f_{1}(k) L}(\overline{a(\bar{k})} A(k)-\lambda \overline{b(\bar{k})} B(k)) \mathcal{B}(k)-(a(k) B(k)-b(k) A(k)) \mathcal{A}(k)=e^{2 i f_{2}(k) T} c(k),
$$

where $c(k)=c(k, T)$ is an entire function, which is $O\left(\left(1+e^{2 i f_{1}(k) L}\right) / k\right)$ as $k \rightarrow \infty$.

-

$$
g_{l}(0)=\frac{d^{l} q_{0}}{d x^{l}}(0), \quad h_{l}(0)=\frac{d^{l} q_{0}}{d x^{l}}(L), \quad 0 \leq l \leq n-1
$$

- for the $s G: q_{1}(0)=g_{0}^{\prime}(0), \quad q_{1}(L)=h_{0}^{\prime}(0)$

Then we call the functions $\left\{g_{l}(t)\right\}_{0}^{n-1},\left\{h_{l}(t)\right\}_{0}^{n-1}$ an admissible set of functions with respect to $q_{0}(x)$ $\left(q_{0}(x)\right.$ and $q_{1}(x)$ for the $s G$ equation).

The main result on the inverse spectral problem is the following:

Theorem 1. Let $q_{0}(x)$ be a smooth function (for the $s G$, let $q_{j}(x), j=0,1$, be smooth functions). Suppose that the set of functions $\left\{g_{l}(t)\right\}_{0}^{n-1},\left\{h_{l}(t)\right\}_{0}^{n-1}$ is admissible with respect to $q_{0}(x)\left(q_{0}(x)\right.$ and $\left.q_{1}(x)\right)$.

Define $M(x, t, k)$ as the solution of the following $2 \times 2$ matrix $R H$ problem:

- $M$ is sectionally holomorphic in $k \in \mathbb{C} \backslash \Sigma$.

- For $k \in \Sigma, M$ satisfies the jump conditions (2.38), where the jump matrix $J$ is defined in terms of the spectral functions $a, b, A, B, \mathcal{A}$ and $\mathcal{B}$ by equations (2.41) - (2.43).

- As $k \rightarrow \infty$,

$$
M(x, t, k)=I+O\left(\frac{1}{k}\right)
$$

Then:

1. $M(x, t, k)$ exists and is unique;

2. The function $q(x, t)$ defined in terms of $M(x, t, k)$ by

$$
\begin{aligned}
q(x, t) & =2 i \lim _{k \rightarrow \infty} k(M(x, t, k))_{12}, \\
q(x, t) & =-2 i \lim _{k \rightarrow \infty} k(M(x, t, k))_{12}, \\
\cos q(x, t) & =1+2 \lim _{k \rightarrow \infty}\left\{k(M(x, t, k))_{12}^{2}+2 i \partial_{x} k(M(x, t, k))_{22}\right\}
\end{aligned}
$$

satisfies the $M K d V I$, the $M K d V I I$, and the $s G$, respectively;

3. The function $q(x, t)$ satisfies the initial and boundary conditions

$$
\begin{aligned}
q(x, 0) & =q_{0}(x) \quad\left(\text { and } q_{t}(x, 0)=q_{1}(x) \text { for the } S G\right), \\
\left\{\partial_{x}^{l} q(0, t)\right. & \left.=g_{l}(t)\right\}_{0}^{n-1}, \quad\left\{\partial_{x}^{l} q(L, t)=h_{l}(t)\right\}_{0}^{n-1},
\end{aligned}
$$


Proof. The proof follows the same lines as in the case of nonlinear integrable equations on the half-line see [6]; for the more detailed presentation, see [5] for the case of the NLS equation. The main steps of the proof include the following.

1. The $\mathrm{RH}$ problem in question is regular; its unique solvability is a consequence of a "vanishing lemma" for the associated RH problem with the vanishing condition at infinity $M=O(1 / k), k \rightarrow \infty$ (see [14]).

2. The proof that the constructed $q(x, t)$ solves the $\mathrm{mKdV}$ equation is straightforward and follows the proof (the so-called dressing method) in the case of a whole line problem, see [15].

3. The proof that $q$ satisfies the initial condition $q(x, 0)=q_{0}(x)$ follows from the fact that it is possible to map the RH problem for $M(x, 0, k)$ to that for $M^{(x)}(x, k)$ such that

$$
M^{(x)}(x, k)=M(x, 0, k) P^{(x)}(x, k)
$$

where $P^{(x)}(x, k)$ is piecewise holomorphic relative to $\Sigma$ and $P^{(x)}(x, k)=I+O\left(\frac{1}{k}\right)$ as $k \rightarrow \infty$.

From (2.27) and (2.39) it follows that

$$
P^{(x)}(x, k)=\left\{\begin{array}{cc}
\left(\begin{array}{cc}
1-\rho \overline{\Gamma(\bar{k})} e^{-2 i f_{1}(k) x} \\
0 & 1
\end{array}\right), & k \in D_{3} \backslash \bar{D}_{0} \\
\left(\begin{array}{cc}
1 & 1 \\
-\rho \overline{\Gamma_{1}(\bar{k})} e^{2 i f_{1}(k) x} & 1
\end{array}\right), & k \in D_{4} \backslash \bar{D}_{0} \\
\left(\begin{array}{cc}
1 & 0 \\
-\Gamma(k) e^{2 i f_{1}(k) x} & 1
\end{array}\right), & k \in D_{2} \backslash \bar{D}_{0} \\
\left(\begin{array}{cc}
1 & -\Gamma_{1}(k) e^{-2 i f_{1}(k) x} \\
0 & 1
\end{array}\right), & k \in D_{1} \backslash \bar{D}_{0}
\end{array}\right.
$$

Now (2.24) and (2.25) imply that $\overline{\Gamma(\bar{k})}$ is $O\left(\frac{1}{k}\right)$ in $D_{3}$ and $\overline{\Gamma_{1}(\bar{k})} e^{2 i f_{1}(k) x}=O\left(\frac{1}{k}\right) e^{-2 i f_{1}(k)(L-x)}$ in $D_{4}$, respectively. Similarly, $\Gamma(k)$ is $O\left(\frac{1}{k}\right)$ in $D_{2}$ and $\Gamma_{1}(k) e^{-2 i f_{1}(k) x}=O\left(\frac{1}{k}\right) e^{2 i f_{1}(k)(L-x)}$ in $D_{1}$, respectively. Therefore, $P^{(x)}(x, k)=I+P_{o f f}^{(x)}(x, k)$, where $P_{o f f}^{(x)}(x, k)$ is off- diagonal and exponentially decaying as $k \rightarrow \infty$ for $\operatorname{Im} k \neq 0$. Finally, the asymptotics (3.4) and (3.13) for $t=0$ yield $q_{0}(x)=q(x, 0)$.

4. The proof that $q$ satisfies the boundary conditions $\left\{\partial_{x}^{l} q(0, t)=g_{l}(t)\right\}_{0}^{n-1}$ and $\left\{\partial_{x}^{l} q(L, t)=h_{l}(t)\right\}_{0}^{n-1}$ is, in turn, based on the maps $M(0, t, k) \mapsto M^{(t)}(t, k)$ and $M(L, t, k) \mapsto M_{1}^{(t)}(t, k)$ :

$$
M^{(t)}(t, k)=M(0, t, k) P^{(t)}(t, k), \quad M_{1}^{(t)}(t, k)=M(L, t, k) P_{1}^{(t)}(t, k) .
$$

In this case, the fact that $P^{(t)}$ and $P_{1}^{(t)}$ in the corresponding domains are $I+O\left(\frac{1}{k}\right)$, is a consequence of the global relation. Consider, for instance, the domain $\operatorname{Im} f_{1}(k)<0$ (considerations in the domain $\operatorname{Im} f_{1}(k)>0$ are similar). We have

$$
P^{(t)}(t, k)=\left\{\begin{array}{cc}
\left(\begin{array}{cc}
\frac{1}{\overline{d(\bar{k})}} & 0 \\
-\rho \frac{\overline{b(\bar{k})}}{A(k)} e^{2 i f_{2}(k) t} & \overline{d(\bar{k})}
\end{array}\right), & k \in D_{3} \backslash \bar{D}_{0} \\
\left(\begin{array}{cc}
\overline{\frac{A(\bar{k})}{\overline{a(\bar{k})}}} & 0 \\
-\frac{\overline{(G R)(\bar{k}) a(\bar{k})}}{\overline{d_{1}(\bar{k})}} e^{2 i f_{2}(k) t} & \frac{\overline{a(\bar{k})}}{\overline{A(\bar{k})}}
\end{array}\right), \quad k \in D_{4} \backslash \bar{D}_{0}
\end{array}\right.
$$


where $(G R)(k)$ is the l.h.s. of the global relation (1.9). Therefore, it is the global relation (1.9) for $k \in D_{1}$ that gives $\overline{(G R)(\bar{k})} e^{2 i f_{2}(k) T}=O\left(\frac{1}{k}\right)$ for $k \in D_{4}\left(D_{4}\right.$ is symmetric to $D_{1}$ under complex conjugation) and, consequently, $P_{21}^{(t)}(t, k)=O\left(\frac{1}{k}\right)$ for $k \in D_{4}$ and $t<T$.

The transformation matrix $P_{1}^{(t)}(t, k)$ is written as

$$
P_{1}^{(t)}(t, k)=\left\{\begin{array}{cc}
\left(\begin{array}{cc}
\frac{1}{A_{1}(k)} & \frac{(G R)(k)}{\overline{d(\bar{k})}} e^{-2 i\left(f_{1}(k) L+f_{2}(k) t\right)} \\
0 & A_{1}(k)
\end{array}\right), \quad k \in D_{3} \backslash \bar{D}_{0} \\
\left(\begin{array}{cc}
\frac{\overline{d_{1}(\bar{k})}}{\overline{a(\bar{k})}} & \frac{b(k)}{\overline{a(\bar{k}) A_{1}(\bar{k})}} e^{-2 i\left(f_{1}(k) L+f_{2}(k) t\right)} \\
0 & \frac{\overline{a(\bar{k})}}{\overline{d_{1}(\bar{k})}}
\end{array}\right), \quad k \in D_{4} \backslash \bar{D}_{0}
\end{array}\right.
$$

Now (1.9) in $D_{3}$ gives

$$
(G R)(k) e^{-2 i\left(f_{1}(k) L+f_{2}(k) T\right)}=O\left(\frac{1}{k}\right),
$$

hence, $\left(P_{1}^{(t)}\right)_{12}(t, k)=O\left(\frac{1}{k}\right)$ for $t<T$ and $k \in D_{3}$. The boundedness of $\left(P_{1}^{(t)}\right)_{12}$ in $D_{4}$ follows from (2.24).

Finally, we obtain that $P^{(t)}(t, k)$ is bounded and piecewise analytic and $P^{(t)}(t, k)=P_{\text {diag }}^{(t)}(t, k)+$ $P_{\text {off }}^{(t)}(t, k)$, where $P_{\text {diag }}^{(t)}(t, k)$ is a diagonal matrix and $P_{o f f}^{(t)}(t, k)$ is off-diagonal and exponentially decaying as $k \rightarrow \infty$, and $P_{\text {diag }}^{(t)}=I+O\left(\frac{1}{k}\right)$. Similarly, $P_{1}^{(t)}(t, k)=\left(P_{1}^{(t)}\right)_{\text {diag }}(t, k)+\left(P_{1}^{(t)}\right)_{\text {off }}(t, k)$ with the same properties. Now recall that in the dressing method, one obtains expressions for $\partial_{x}^{l} q(x, t)$ in terms of $M(x, t, k)$. Since these expressions evaluated at $x=0$ are Eqs. (3.8) with $M^{(t)}$ replaced by $M(0, t, k)$ (similarly for $x=L$ and $M(L, t, k)$ ), the maps (3.17) provide the boundary conditions at $x=0$ and $x=L$. Alternatively, one can use the fact that the multiplication by a diagonal matrix does not affect the r.h.s. of (3.8).

\section{Analysis of the global relation}

The analysis of the global relation consists in the following. First, we consider (2.36) as a family of relations parametrized by $t \in(0, T)$, with $A=A(t, k), B=B(t, k), \mathcal{A}=\mathcal{A}(t, k), \mathcal{B}=\mathcal{B}(t, k)$, and $c=c(t, k)$. Then, we substitute the spectral functions $A(t, k), B(t, k), \mathcal{A}(t, k), \mathcal{B}(t, k)$ in (2.36) by their integral representations in the form

$$
\begin{aligned}
& A(t, k)=1+2 \int_{0}^{t} \overline{F_{2}(t, t-2 \tau, \bar{k})} e^{2 i f_{2}(k) \tau} d \tau, \\
& B(t, k)=-2 \int_{0}^{t} F_{1}(t, 2 \tau-t, k) e^{2 i f_{2}(k) \tau} d \tau
\end{aligned}
$$

(with similar expressions for $\mathcal{A}(t, k)$ and $\mathcal{B}(t, k)$ ), where $F_{1}(t, s, k)$ and $F_{2}(t, s, k)$ are polynomials (in the case of the $\mathrm{mKdV}$ equations) or rational functions (in the case of the $\mathrm{sG}$ ) in $k$ with coefficients satisfying a linear system of differential equations determined by the boundary values $\left\{g_{l}(t)\right\}_{0}^{n-1}$ and $\left\{h_{l}(t)\right\}_{0}^{n-1}$. Further, we use certain symmetries of $A(t, k), B(t, k), \mathcal{A}(t, k), \mathcal{B}(t, k)$ and integrate the resulting expressions (multiplied by appropriate exponentials) over the boundary of some domain in the $k$-plane. Finally, assuming that a set of boundary values corresponding to some well-posed problem is given, we resolve the obtained system of equations in terms of the unknown boundary values.

sG: 
Lemma 1. The spectral functions $A(t, k), B(t, k), \mathcal{A}(t, k), \mathcal{B}(t, k)$ have the following integral representations

$$
\begin{aligned}
A(t, k) & =1+2 \int_{0}^{t} \overline{F_{2}(t, t-2 \tau, \bar{k})} e^{\frac{i}{2}\left(k+\frac{1}{k}\right) \tau} d \tau, \\
B(t, k) & =-2 \int_{0}^{t} F_{1}(t, 2 \tau-t, k) e^{\frac{i}{2}\left(k+\frac{1}{k}\right) \tau} d \tau, \\
\mathcal{A}(t, k) & =1+2 \int_{0}^{t} \overline{\mathcal{F}_{2}(t, t-2 \tau, \bar{k})} e^{\frac{i}{2}\left(k+\frac{1}{k}\right) \tau} d \tau, \\
\mathcal{B}(t, k) & =-2 \int_{0}^{t} \mathcal{F}_{1}(t, 2 \tau-t, k) e^{\frac{i}{2}\left(k+\frac{1}{k}\right) \tau} d \tau .
\end{aligned}
$$

Here $F_{1}$ and $F_{2}$ are polynomials in $1 / k$ of degree 1 :

$$
\begin{aligned}
F_{1}(t, s, k) & =\frac{1}{k} F_{1}^{(1)}(t, s)+F_{1}^{(0)}(t, s) \\
& \equiv-\frac{1}{2 k}\left[M_{1}(t, s)+\tan \frac{g_{0}(t)}{2} M_{2}(t, s)\right]+i L_{1}(t, s), \\
F_{2}(t, s, k) & =\frac{1}{k} F_{2}^{(1)}(t, s)+F_{2}^{(0)}(t, s) \\
& \equiv-\frac{i}{2 k}\left[-M_{2}(t, s)+\tan \frac{g_{0}(t)}{2} M_{1}(t, s)\right]+L_{2}(t, s),
\end{aligned}
$$

where $L_{j}(t, s)$ and $M_{j}(t, s), j=1,2$ solve the Goursat problem for a certain system of linear differential equations in the domain $0<t<T,-t<s<t$ with coefficients determined by $g_{0}(t)$ and $g_{1}(t)$ and with the boundary conditions

$$
\begin{aligned}
L_{1}(t, t) & =-\frac{1}{8}\left(\dot{g}_{0}(t)+g_{1}(t)\right), & L_{2}(t,-t) & =0, \\
M_{1}(t, t) & =-\frac{1}{4} \sin g_{0}(t), & M_{2}(t,-t) & =0 .
\end{aligned}
$$

Analogously, the functions $\mathcal{F}_{1}$ and $\mathcal{F}_{2}$ are expressed in terms of $\mathcal{L}_{j}$ and $\mathcal{M}_{j}$ satisfying the similar system of equations, with $\left\{g_{l}(t)\right\}_{0}^{1}$ replaced by $\left\{h_{l}(t)\right\}_{0}^{1}$; particularly,

$$
\begin{aligned}
\mathcal{L}_{1}(t, t) & =-\frac{1}{8}\left(\dot{g}_{0}(t)+g_{1}(t)\right), & \mathcal{L}_{2}(t,-t) & =0, \\
\mathcal{M}_{1}(t, t) & =-\frac{1}{4} \sin g_{0}(t), & \mathcal{M}_{2}(t,-t) & =0 .
\end{aligned}
$$

The proof of the lemma is based on the Gelfand-Levitan-Marchenko representation for the solution of the $t$-equation in the Lax pair (1.7) with the initial condition $\mu(0, k)=I$ :

$$
\mu(t, k)=I+\int_{-t}^{t}\left(L(t, s)+\frac{1}{k} M(t, s)\right) e^{\frac{i}{4}\left(k+\frac{1}{k}\right)(t-s) \sigma_{3}} d s,
$$

the distinctive feature of which is the explicit $k$-dependence. The functions $L_{j}$ and $M_{j}$ are simply expressed in terms of the matrix entries of $L$ and $M$. For the NLS equation, the corresponding representation is presented in details in [8]. For the sG equation, the details are given in [9].

To avoid routine technical complications, we will continue the analysis of the global relation in the case $a(k) \equiv 1, b(k) \equiv 0$ corresponding to the zero initial conditions $q(x, 0)=q_{t}(x, 0)=0$. In this case, the global relation (2.36) takes the form

$$
e^{\frac{i}{2}\left(k-\frac{1}{k}\right) L} A(t, k) \mathcal{B}(t, k)-B(t, k) \mathcal{A}(t, k)=e^{\frac{i}{2}\left(k+\frac{1}{k}\right) t} c(t, k) .
$$

From (2.37) and (2.7d) it follows that

$$
c(t, k)=O\left(\frac{1}{k}\right) \quad \text { as } k \rightarrow \infty, \quad k \in D_{1}=\{k: \operatorname{Im} k \geq 0,|k| \geq 1\}
$$


and $e^{-\frac{i}{2}\left(k-\frac{1}{k}\right) L} c(t, k)$ is bounded in $D_{3}=\{k: \operatorname{Im} k \leq 0,|k| \leq 1\}$.

In the case of the sG equation, the given (linearly well-posed) boundary conditions are $g_{0}(t)$ and $h_{0}(t)$, and we are looking for expressions for $g_{1}(t)$ and $h_{1}(t)$.

Substitute (4.2) into (4.6) and rewrite the resulting equation in the form:

$$
\begin{aligned}
-e^{\frac{i}{2}\left(k-\frac{1}{k}\right) L} \int_{0}^{t} \mathcal{F}_{1}^{(0)}(t, 2 \tau-t) e^{\frac{i}{2}\left(k+\frac{1}{k}\right) \tau} d \tau & +\int_{0}^{t} F_{1}^{(0)}(t, 2 \tau-t) e^{\frac{i}{2}\left(k+\frac{1}{k}\right) \tau} d \tau= \\
G(t, k) & +\frac{1}{2} e^{\frac{i}{2}\left(k+\frac{1}{k}\right) t} c(t, k)
\end{aligned}
$$

where

$$
\begin{aligned}
G(t, k)= & e^{\frac{i}{2}\left(k-\frac{1}{k}\right) L}\left(\frac{1}{k} \int_{0}^{t} \mathcal{F}_{1}^{(1)}(t, 2 \tau-t) e^{\frac{i}{2}\left(k+\frac{1}{k}\right) \tau} d \tau\right. \\
& \left.+2 \int_{0}^{t} \overline{F_{2}(t, t-2 \tau, \bar{k})} e^{\frac{i}{2}\left(k+\frac{1}{k}\right) \tau} d \tau \int_{0}^{t} \mathcal{F}_{1}(t, t-2 \tau, k) e^{\frac{i}{2}\left(k+\frac{1}{k}\right) \tau} d \tau\right) \\
& -\frac{1}{k} \int_{0}^{t} F_{1}^{(1)}(t, 2 \tau-t) e^{\frac{i}{2}\left(k+\frac{1}{k}\right) \tau} d \tau \\
& -2 \int_{0}^{t} \overline{\mathcal{F}_{2}(t, t-2 \tau, \bar{k})} e^{\frac{i}{2}\left(k+\frac{1}{k}\right) \tau} d \tau \int_{0}^{t} F_{1}(t, t-2 \tau, k) e^{\frac{i}{2}\left(k+\frac{1}{k}\right) \tau} d \tau
\end{aligned}
$$

We consider (4.8) in the domain $D_{1}=\{k: \operatorname{Im} k \geq 0,|k| \geq 1\}$ where all the terms of (4.8) are bounded. In order to obtain another equation with terms bounded in $D_{1}$, we replace $k$ by $1 / k$ in (4.8) and multiply by $e^{\frac{i}{2}\left(k-\frac{1}{k}\right) L}$; the resulting equation is

$$
\begin{aligned}
-\int_{0}^{t} \mathcal{F}_{1}^{(0)}(t, 2 \tau-t) e^{\frac{i}{2}\left(k+\frac{1}{k}\right) \tau} d \tau & +e^{\frac{i}{2}\left(k-\frac{1}{k}\right) L} \int_{0}^{t} F_{1}^{(0)}(t, 2 \tau-t) e^{\frac{i}{2}\left(k+\frac{1}{k}\right) \tau} d \tau= \\
e^{\frac{i}{2}\left(k-\frac{1}{k}\right) L} G\left(t, \frac{1}{k}\right) & +\frac{1}{2} e^{\frac{i}{2}\left(k+\frac{1}{k}\right) t} e^{\frac{i}{2}\left(k-\frac{1}{k}\right) L} c\left(t, \frac{1}{k}\right) .
\end{aligned}
$$

Equations (4.8) and (4.10) can be written in the vector form:

$$
\mathcal{E}(k) U(t, k)=H(t, k)+\frac{1}{2} e^{\frac{i}{2}\left(k+\frac{1}{k}\right) t} H_{c}(t, k), \quad k \in D_{1},
$$

where

$$
\begin{aligned}
& \mathcal{E}(k)=\left(\begin{array}{cc}
-e^{\frac{i}{2}\left(k-\frac{1}{k}\right) L} & 1 \\
-1 & e^{\frac{i}{2}\left(k-\frac{1}{k}\right) L}
\end{array}\right), \\
& U(t, k)=\left(\int_{0}^{t} \mathcal{F}_{1}^{(0)}(t, 2 \tau-t) e^{\frac{i}{2}\left(k+\frac{1}{k}\right) \tau} d \tau \quad \int_{0}^{t} F_{1}^{(0)}(t, 2 \tau-t) e^{\frac{i}{2}\left(k+\frac{1}{k}\right) \tau} d \tau\right)^{T}, \\
& H(t, k)=\left(\begin{array}{c}
G(t, k) \\
e^{\frac{i}{2}\left(k-\frac{1}{k}\right) L} G\left(t, \frac{1}{k}\right)
\end{array}\right), \quad H_{c}(t, k)=\left(\begin{array}{c}
c(t, k) \\
e^{\frac{i}{2}\left(k-\frac{1}{k}\right) L} c\left(t, \frac{1}{k}\right)
\end{array}\right) .
\end{aligned}
$$

Since $\operatorname{det} \mathcal{E}(k)=1-e^{i\left(k-\frac{1}{k}\right) L}, \mathcal{E}^{-1}(k)$ is bounded in the domain $D_{1}^{(0)} \subset D_{1}$, the boundary of which is the boundary of $D_{1}$ deformed toward the interior of $D_{1}$ such that it passes above the zeros of $\operatorname{det} \mathcal{E}(k)$ : $k=\frac{\pi n}{L} \pm \sqrt{\frac{\pi^{2} n^{2}}{L^{2}}+1}, n \in \mathbb{Z}$.

Multiplying (4.11) by $\mathcal{E}^{-1}(k)\left(1-\frac{1}{k^{2}}\right) e^{-\frac{i}{2}\left(k+\frac{1}{k}\right) t^{\prime}}, 0<t^{\prime}<t$, and integrating over the contour $\partial D_{1}^{(0)}$, we obtain the following equation:

$$
\begin{aligned}
& \int_{\partial D_{1}^{(0)}} U(t, k)\left(1-\frac{1}{k^{2}}\right) e^{-\frac{i}{2}\left(k+\frac{1}{k}\right) t^{\prime}} d k= \\
& \int_{\partial D_{1}^{(0)}} \mathcal{E}^{-1}(k)\left(1-\frac{1}{k^{2}}\right)\left(H(t, k)+\frac{1}{2} e^{\frac{i}{2}\left(k+\frac{1}{k}\right) t} H_{c}(t, k)\right) e^{-\frac{i}{2}\left(k+\frac{1}{k}\right) t^{\prime}} d k
\end{aligned}
$$


Using the identity

$$
\int_{\partial D_{1}^{(0)}}\left(1-\frac{1}{k^{2}}\right) \int_{0}^{t} \alpha(\tau) e^{\frac{i}{2}\left(k+\frac{1}{k}\right)\left(\tau-t^{\prime}\right)} d t d k=4 \pi \alpha\left(t^{\prime}\right), \quad 0<t^{\prime}<t
$$

valid for any smooth function $\alpha(\tau), \tau \in[0, t]$ (which follows from the usual Fourier transform identity), the left-hand side of (4.12) becomes

$$
4 \pi\left(\mathcal{F}_{1}^{(0)}\left(t, 2 t^{\prime}-t\right) \quad F_{1}^{(0)}\left(t, 2 t^{\prime}-t\right)\right)^{T} .
$$

Since $c(t, k)=O(1 / k)$ as $k \rightarrow \infty$ in $D_{1}$, the terms in the right-hand side of (4.12) involving $c(t, k)$, vanish (by Jordan's Lemma). The integration by parts shows that $G(t, k)=O(1 / k)$ on $\partial D_{1}^{(0)}$, so that the integrals involving $G(t, k)$ exist in the $L^{2}$ sense. On the other hand, the functions $c(t, 1 / k)$ and $G(t, 1 / k)$ do not vanish as $k \rightarrow \infty$. The following lemma describes the behavior of $c(t, k)$ and $G(t, k)$ as $k \rightarrow 0$ for $\operatorname{Im} k \leq 0$, which in turn gives the behavior of $c(t, 1 / k)$ and $G(t, 1 / k)$ as $k \rightarrow \infty$ in $D_{1}$.

Lemma 2. The following asymptotics hold as $k \rightarrow 0$ in $\operatorname{Im} k \leq 0$ :

$$
\begin{gathered}
e^{-\frac{i}{2}\left(k-\frac{1}{k}\right) L} G(t, k)=e^{\frac{i}{2 k} t}\left(-\alpha(t)+e^{\frac{i}{2 k} L} \beta(t)\right)+O(k) \\
\frac{1}{2} e^{-\frac{i}{2}\left(k-\frac{1}{k}\right) L} c(t, k)=\alpha(t)-e^{\frac{i}{2 k} L} \beta(t)+O(k),
\end{gathered}
$$

where

$$
\begin{aligned}
& \alpha(t)=\frac{i}{2} \sin \frac{h_{0}(t)}{2} \cos \frac{g_{0}(t)}{2}, \\
& \beta(t)=\frac{i}{2} \sin \frac{g_{0}(t)}{2} \cos \frac{h_{0}(t)}{2} .
\end{aligned}
$$

Proof. (i) By the definition, $G(t, k)=\frac{1}{2}\left(B(t, k) \mathcal{A}(t, k)-e^{\frac{i}{2}\left(k-\frac{1}{k}\right) L} A(t, k) \mathcal{B}(t, k)\right)+O(k), k \rightarrow 0$. From (2.15) and (2.16) it follows that $A, B$ and $\mathcal{A}, \mathcal{B}$ can be expressed via the second columns of $\mu_{1}(0,0, k)$ and $\mu_{4}(0,0, k)$, respectively:

$$
\left(\begin{array}{l}
B(T, k) \\
A(T, k)
\end{array}\right)=\mu_{1}^{(2)}(0,0, k), \quad\left(\begin{array}{c}
\mathcal{B}(T, k) \\
\mathcal{A}(T, k)
\end{array}\right)=\mu_{4}^{(2)}(0,0, k) .
$$

In order to study the small $k$ behavior of $\mu_{1}^{(2)}(0,0, k)$ we notice that $\tilde{Q}$ in $(2.1 \mathrm{~b})$ can be written as

$$
\tilde{Q}(x, t, k)=-\frac{i}{4}\left(q_{x}(x, t)+q_{t}(x, t)\right) \sigma_{1}+\frac{i}{4 k}\left(\sigma_{3}-\Lambda^{-1}(x, t) \sigma_{3} \Lambda(x, t)\right),
$$

where

$$
\Lambda(x, t)=\left(\begin{array}{cc}
1+\cos q(x, t) & i \sin q(x, t) \\
i \sin q(x, t) & 1+\cos q(x, t)
\end{array}\right) .
$$

This suggests to represent $\mu_{1}(0, t, k), 0 \leq t \leq T$ in the form

$$
\mu_{1}(0, t, k)=\Lambda^{-1}(0, t) \nu_{1}(t, k) e^{-\frac{i}{4}\left(k+\frac{1}{k}\right) \sigma_{3}(t-T)} \Lambda(0, T) e^{\frac{i}{4}\left(k+\frac{1}{k}\right) \sigma_{3}(t-T)},
$$

where $\nu_{1}(t, k)$ satisfies a differential $t$-equation similar to that for $\mu_{1}(0, t, k)$, see (1.7), but with the right-hand side which is non-singular at $k=0$ :

$$
\frac{d \nu_{1}}{d t}+\frac{i}{4}\left(k+\frac{1}{k}\right) \hat{\sigma}_{3} \nu_{1}=\hat{Q}(t, k) \nu_{1}
$$

where

$$
\hat{Q}(t, k)=-\frac{i k}{4}\left(\begin{array}{cc}
-1+\cos g_{0}(t) & -i \sin g_{0}(t) \\
i \sin g_{0}(t) & 1-\cos g_{0}(t)
\end{array}\right)-\frac{g_{0}^{\prime}(t)}{2} \sin g_{0}(t) I-\frac{i}{4}\left(g_{1}(t)-g_{0}^{\prime}(t)\right) \sigma_{1}
$$


and $\nu_{1}(T, k)=I$. Now the small $k$ behavior of $\nu_{1}(t, k)$ can be easily obtained from the analysis of the corresponding Volterra integral equations satisfied by the columns of $\nu_{1}(t, k)$. More precisely, since

$$
\mu_{1}^{(2)}(0, t, k)=\Lambda^{-1}(0, t)\left(\nu_{1}^{(1)}(t, k) e^{-\frac{i}{2}\left(k+\frac{1}{k}\right)(t-T)} \nu_{1}^{(2)}(t, k)\right) \Lambda^{(2)}(0, T),
$$

we need the Volterra integral equations for $\tilde{\nu}_{1}^{(1)}:=\nu_{1}^{(1)} e^{-\frac{i}{2}\left(k+\frac{1}{k}\right)(t-T)}$ and $\nu_{2}$. From (4.13) we deduce the integral equations

$$
\tilde{\nu}_{1}^{(1)}(t, k)=\left(\begin{array}{c}
e^{-\frac{i}{2}\left(k+\frac{1}{k}\right)(t-T)} \\
0
\end{array}\right)-\int_{t}^{T}\left(\begin{array}{cc}
e^{-\frac{i}{2}\left(k+\frac{1}{k}\right)(t-\tau)} & 0 \\
0 & 1
\end{array}\right) \hat{Q}(\tau, k) \tilde{\nu}_{1}^{(1)}(\tau, k) d \tau
$$

and

$$
\nu_{1}^{(2)}(t, k)=\left(\begin{array}{l}
0 \\
1
\end{array}\right)-\int_{t}^{T}\left(\begin{array}{cc}
e^{-\frac{i}{2}\left(k+\frac{1}{k}\right)(t-\tau)} & 0 \\
0 & 1
\end{array}\right) \hat{Q}(\tau, k) \nu_{1}^{(2)}(\tau, k) d \tau
$$

from which we conclude that

$$
\begin{aligned}
& \tilde{\nu}_{1}^{(1)}(t, k)=\left(\frac{\cos \frac{g_{0}(t)}{2}}{\cos \frac{g_{0}(T)}{2}}\right) e^{-\frac{i}{2}\left(k+\frac{1}{k}\right)(t-T)}+O(k), \\
& \nu_{1}^{(2)}(t, k)=\left(\begin{array}{c}
0 \\
\cos \frac{g_{0}(t)}{2} \\
\cos \frac{g_{0}(T)}{2}
\end{array}\right)+O(k)
\end{aligned}
$$

as $k \rightarrow 0, \operatorname{Im} k \leq 0$.

Finally, combining (4.14) and (4.15) evaluated at $t=0$, we obtain

$$
\left(\begin{array}{c}
B(T, k) \\
A(T, k)
\end{array}\right)=\left(\begin{array}{c}
i \sin \frac{g_{0}(T)}{2} \cos \frac{g_{0}(0)}{2} e^{\frac{i}{2 k} T}-i \sin \frac{g_{0}(0)}{2} \cos \frac{g_{0}(T)}{2} \\
\sin \frac{g_{0}(0)}{2} \sin \frac{g_{0}(T)}{2}+\cos \frac{g_{0}(0)}{2} \cos \frac{g_{0}(T)}{2}
\end{array}\right)+O(k), \quad k \rightarrow 0, \quad \operatorname{Im} k \leq 0 .
$$

Particularly, in the case of $g_{0}(0)=0$, we have (replacing $T$ by $t$ )

$$
B(t, k)=i \sin \frac{g_{0}(t)}{2} \exp \left\{\frac{i}{2 k} t\right\}+O(k), \quad A(t, k)=\cos \frac{g_{0}(t)}{2}+O(k) .
$$

Similarly,

$$
\mathcal{B}(t, k)=i \sin \frac{h_{0}(t)}{2} \exp \left\{\frac{i}{2 k} t\right\}+O(k), \quad \mathcal{A}(t, k)=\cos \frac{h_{0}(t)}{2}+O(k),
$$

and statement (i) of the lemma follows.

(ii) Since $c(T, k)=\left(\mu_{4}\right)_{12}(0, T, k)$, we need to study the small $k$ behavior of $\mu_{4}(0, T, k)$. The analysis follows the same lines as in the proof of (i). Namely, we notice that $Q$ in (2.1a) can be written as

$$
Q(x, t, k)=-\frac{i}{4}\left(q_{x}(x, t)+q_{t}(x, t)\right) \sigma_{1}-\frac{i}{4 k}\left(\sigma_{3}-\Lambda^{-1}(x, t) \sigma_{3} \Lambda(x, t)\right),
$$

which suggests to represent $\mu_{4}(x, T, k), 0 \leq x \leq L$ in the form

$$
\mu_{4}(x, T, k)=\Lambda^{-1}(x, T) \nu_{4}(x, k) e^{-\frac{i}{4}\left(k-\frac{1}{k}\right) \sigma_{3}(x-L)} \Lambda(L, T) e^{\frac{i}{4}\left(k-\frac{1}{k}\right) \sigma_{3}(x-L)},
$$

where $\nu_{4}(x, k)$ satisfies the $x$-differential equation

$$
\frac{d \nu_{4}}{d x}+\frac{i}{4}\left(k-\frac{1}{k}\right) \hat{\sigma}_{3} \nu_{4}=\check{Q}(x, k) \nu_{4}
$$

with

$$
\check{Q}(x, k)=-\frac{i k}{4}\left(\begin{array}{cc}
-1+\cos q(x, T) & -i \sin q(x, T) \\
i \sin q(x, T) & 1-\cos q(x, T)
\end{array}\right)-\frac{q_{x}(x, T)}{2} \sin q(x, T) I+\frac{i}{4}\left(q_{x}(x, T)-q_{t}(x, T)\right) \sigma_{1} .
$$


From (4.16) we have

$$
\mu_{4}^{(2)}(x, T, k) e^{-\frac{i}{2}\left(k-\frac{1}{k}\right)(L-x)}=\Lambda^{-1}(x, T)\left(\nu_{4}^{(1)}(x, k) \quad \nu_{4}^{(1)}(x, k) e^{-\frac{i}{2}\left(k-\frac{1}{k}\right)(L-x)}\right) \Lambda^{(2)}(L, T) .
$$

Equation (4.17) together with the initial condition $\nu_{4}(L, k)=I$ leads to the Volterra integral equations for $\nu_{4}^{(1)}$ and $\tilde{\nu}_{4}^{(2)}:=\nu_{4}^{(2)} e^{-\frac{i}{4}\left(k-\frac{1}{k}\right)(L-x)}$ :

$$
\begin{gathered}
\nu_{4}^{(1)}(x, k)=\left(\begin{array}{l}
1 \\
0
\end{array}\right)-\int_{x}^{L}\left(\begin{array}{cc}
1 & 0 \\
0 & e^{-\frac{i}{2}\left(k-\frac{1}{k}\right)(y-x)}
\end{array}\right) \check{Q}(x, y) \nu_{4}^{(1)}(y, k) d y \\
\tilde{\nu}_{4}^{(2)}(x, k)=\left(\begin{array}{c}
0 \\
e^{-\frac{i}{2}\left(k-\frac{1}{k}\right)(L-x)}
\end{array}\right)-\int_{x}^{L}\left(\begin{array}{cc}
1 & 0 \\
0 & e^{-\frac{i}{2}\left(k-\frac{1}{k}\right)(y-x)}
\end{array}\right) \check{Q}(x, y) \tilde{\nu}_{4}^{(2)}(y, k) d y,
\end{gathered}
$$

from which we conclude that

$$
\begin{aligned}
& \nu_{4}^{(1)}(x, k)=\left(\frac{\frac{\cos \frac{q(x, T)}{2}}{\cos \frac{q(L, T)}{2}}}{0}\right)+O(k), \\
& \tilde{\nu}_{4}^{(2)}(x, k)=\left(\begin{array}{c}
0 \\
\frac{\cos \frac{q(x, T)}{2}}{\cos \frac{q(L, T)}{2}}
\end{array}\right) e^{-\frac{i}{2}\left(k-\frac{1}{k}\right)(L-x)}+O(k)
\end{aligned}
$$

as $k \rightarrow 0, \operatorname{Im} k \leq 0$. Substituting this into (4.18) and evaluating at $x=0$ gives

$$
\left(\mu_{4}\right)_{12}(0, T, k) e^{-\frac{i}{2}\left(k-\frac{1}{k}\right) L}=i \cos \frac{q(0, T)}{2} \sin \frac{q(L, T)}{2}-i \sin \frac{q(0, T)}{2} \cos \frac{q(L, T)}{2} \exp \left\{\frac{i}{2 k} L\right\}+O(k) .
$$

Taking into account that $q(0, T)=g_{0}(T)$ and $q(L, T)=h_{0}(T)$ and replacing $T$ by $t$ we obtain statement (ii). Lemma is proved.

Rewrite the right-hand side of (4.12) as

$$
\int_{\partial D_{1}^{(0)}} \mathcal{E}^{-1}(k)\left(1-\frac{1}{k^{2}}\right) \tilde{H}(t, k) e^{-\frac{i}{2}\left(k+\frac{1}{k}\right) t^{\prime}} d k+\int_{\partial D_{1}^{(0)}} \mathcal{E}^{-1}(k)\left(1-\frac{1}{k^{2}}\right) \tilde{H}_{c}(t, k) e^{\frac{i}{2}\left(k+\frac{1}{k}\right)\left(t-t^{\prime}\right)} d k,
$$

where

$$
\tilde{H}(t, k)=\left(\begin{array}{c}
G(t, k) \\
e^{\frac{i}{2}\left(k-\frac{1}{k}\right) L} G\left(t, \frac{1}{k}\right)+e^{\frac{i}{2}\left(k+\frac{1}{k}\right) t}\left(\alpha(t)-e^{\frac{i k}{2} L} \beta(t)\right)
\end{array}\right)
$$

and

$$
\tilde{H}_{c}(t, k)=\left(\begin{array}{c}
\frac{1}{2} c(t, k) \\
\frac{1}{2} e^{\frac{i}{2}\left(k-\frac{1}{k}\right) L} c\left(t, \frac{1}{k}\right)-\alpha(t)+e^{\frac{i k}{2} L} \beta(t) .
\end{array}\right)
$$

Then Jordan's Lemma together with Lemma 2 implies that the second integral in (4.20) vanishes. Hence, we arrive at the following equation

$$
\left(\begin{array}{l}
\mathcal{F}_{1}^{(0)}\left(t, 2 t^{\prime}-t\right) \\
F_{1}^{(0)}\left(t, 2 t^{\prime}-t\right)
\end{array}\right)=\frac{1}{4 \pi} \int_{\partial D_{1}^{(0)}} \mathcal{E}^{-1}(k)\left(1-\frac{1}{k^{2}}\right) \tilde{H}(t, k) e^{-\frac{i}{2}\left(k+\frac{1}{k}\right) t^{\prime}} d k .
$$

Evaluating this at $t^{\prime}=t$ and using (4.4) and (4.5) in the left-hand side, we find the following equations for $g_{1}(t)$ and $h_{1}(t)$ :

$$
\begin{aligned}
g_{1}(t)= & -\dot{g}_{0}(t)+\frac{2 i}{\pi} \int_{\partial D_{1}^{(0)}} \frac{1}{1-e^{i(k-1 / k) L}}\left(1-\frac{1}{k^{2}}\right)\left\{e ^ { \frac { i } { 2 } ( k - \frac { 1 } { k } ) L } e ^ { - \frac { i } { 2 } ( k + \frac { 1 } { k } ) t } \left(G(t, k)-G\left(t, \frac{1}{k}\right)\right.\right. \\
& \left.-\frac{i}{2} \sin \frac{h_{0}(t)}{2} \cos \frac{g_{0}(t)}{2}+\frac{i}{2} \sin \frac{g_{0}(t)}{2} \cos \frac{h_{0}(t)}{2} e^{\frac{i k}{2} L}\right\}, \\
h_{1}(t)= & -\dot{h}_{0}(t)+\frac{2 i}{\pi} \int_{\partial D_{1}^{(0)}} \frac{1}{1-e^{i(k-1 / k) L}}\left(1-\frac{1}{k^{2}}\right)\left\{e^{-\frac{i}{2}\left(k+\frac{1}{k}\right) t}\left(G(t, k)-e^{i\left(k-\frac{1}{k}\right) L} G\left(t, \frac{1}{k}\right)\right)\right. \\
& \left.-e^{\frac{i}{2}\left(k-\frac{1}{k}\right) L}\left(\frac{i}{2} \sin \frac{h_{0}(t)}{2} \cos \frac{g_{0}(t)}{2}+\frac{i}{2} \sin \frac{g_{0}(t)}{2} \cos \frac{h_{0}(t)}{2} e^{\frac{i k}{2} L}\right)\right\} .
\end{aligned}
$$


The functions $G(t, k)$ and $G\left(t, \frac{1}{k}\right)$ involved in (4.21) can be expressed in terms of $\Phi(t, k)$ and $\Psi(t, k)$, see Definitions 2 and 3. Indeed, (3.6) and (3.9) together with (4.2) give

$$
\begin{aligned}
\int_{0}^{t} F_{1}(t, 2 \tau-t, k) e^{\frac{i}{2}\left(k+\frac{1}{k}\right) \tau} d \tau & =\frac{1}{2} \Phi_{1}(t, k) e^{\frac{i}{2}\left(k+\frac{1}{k}\right) t} \\
\int_{0}^{t} \mathcal{F}_{1}(t, 2 \tau-t, k) e^{\frac{i}{2}\left(k+\frac{1}{k}\right) \tau} d \tau & =\frac{1}{2} \Psi_{1}(t, k) e^{\frac{i}{2}\left(k+\frac{1}{k}\right) t} \\
\int_{0}^{t} \overline{F_{2}(t, t-2 \tau, \bar{k})} e^{\frac{i}{2}\left(k+\frac{1}{k}\right) \tau} d \tau & =\frac{1}{2}\left(\overline{\Phi_{2}(t, \bar{k})}-1\right) \\
\int_{0}^{t} \overline{\mathcal{F}_{2}(t, t-2 \tau, \bar{k})} e^{\frac{i}{2}\left(k+\frac{1}{k}\right) \tau} d \tau & =\frac{1}{2}\left(\overline{\Psi_{2}(t, \bar{k})}-1\right)
\end{aligned}
$$

Since the exponentials in (4.22) depend on $k$ only through $k+1 / k$, supplementing (4.22) with the two equations obtained from (4.22) by replacing $k$ with $1 / k$ and taking into account (4.3) we find the following expressions

$$
\begin{aligned}
\frac{1}{k} \int_{0}^{t} F_{1}^{(1)}(t, 2 \tau-t, k) e^{\frac{i}{2}\left(k+\frac{1}{k}\right) \tau} d \tau & =\frac{1}{2} \frac{1}{1-k^{2}} e^{\frac{i}{2}\left(k+\frac{1}{k}\right) t}\left(\Phi_{1}(t, k)-\Phi_{1}\left(t, \frac{1}{k}\right)\right) \\
\frac{1}{k} \int_{0}^{t} \mathcal{F}_{1}^{(1)}(t, 2 \tau-t, k) e^{\frac{i}{2}\left(k+\frac{1}{k}\right) \tau} d \tau & =\frac{1}{2} \frac{1}{1-k^{2}} e^{\frac{i}{2}\left(k+\frac{1}{k}\right) t}\left(\Psi_{1}(t, k)-\Psi_{1}\left(t, \frac{1}{k}\right)\right)
\end{aligned}
$$

Substituting (4.22)-(4.24) into (4.9) we obtain the following expression for $G$ :

$$
\begin{aligned}
G(t, k)= & \frac{1}{2} e^{\frac{i}{2}\left(k+\frac{1}{k}\right) t}\left\{e^{\frac{i}{2}\left(k-\frac{1}{k}\right) L}\left[\frac{1}{1-k^{2}}\left(\Psi_{1}(t, k)-\Psi_{1}\left(t, \frac{1}{k}\right)\right)+\left(\overline{\Phi_{2}(t, \bar{k})}-1\right) \Psi_{1}(t, k)\right]\right. \\
& \left.-\frac{1}{1-k^{2}}\left(\Phi_{1}(t, k)-\Phi_{1}\left(t, \frac{1}{k}\right)\right)-\left(\overline{\Psi_{2}(t, \bar{k})}-1\right) \Phi_{1}(t, k)\right\} .
\end{aligned}
$$

Using (4.25) in (4.21) we obtain the equations for $g_{1}$ and $h_{1}$ in terms of $\Phi$ and $\Psi$. These equations, together with (3.5) and the similar equation for $\Psi(t, k)$ constitute a system of four nonlinear ODEs for $\Phi_{1}, \Phi_{2}, \Psi_{1}$, and $\Psi_{2}$, which in turn is equivalent to four nonlinear integral equations of the Volterra type.

\section{mKdV I:}

Lemma 3. The spectral functions $A(t, k), B(t, k), \mathcal{A}(t, k), \mathcal{B}(t, k)$ have the following integral representations

$$
\begin{aligned}
& A(t, k)=1+2 \int_{0}^{t} \overline{F_{2}(t, t-2 \tau, \bar{k})} e^{8 i k^{3} \tau} d \tau, \\
& B(t, k)=-2 \int_{0}^{t} F_{1}(t, 2 \tau-t, k) e^{8 i k^{3} \tau} d \tau \\
& \mathcal{A}(t, k)=1+2 \int_{0}^{t} \overline{\mathcal{F}_{2}(t, t-2 \tau, \bar{k})} e^{8 i k^{3} \tau} d \tau \\
& \mathcal{B}(t, k)=-2 \int_{0}^{t} \mathcal{F}_{1}(t, 2 \tau-t, k) e^{8 i k^{3} \tau} d \tau .
\end{aligned}
$$


Here $F_{1}$ and $F_{2}$ are polynomials in $k$ of degree 2 :

$$
\begin{aligned}
F_{1}(t, s, k)= & k^{2} F_{1}^{(2)}(t, s)+i k F_{1}^{(1)}(t, s)+F_{1}^{(0)}(t, s) \\
\equiv & k^{2} N_{1}(t, s)+i k\left(M_{1}(t, s)-\frac{1}{2} g_{0}(t) N_{2}(t, s)\right) \\
& \left.+L_{1}(t, s)+\frac{1}{2} g_{0}(t) M_{2}(t, s)\right)+\frac{1}{4} g_{1}(t) N_{2}(t, s), \\
F_{2}(t, s, k)= & k^{2} F_{2}^{(2)}(t, s)+i k F_{2}^{(1)}(t, s)+F_{2}^{(0)}(t, s) \\
\equiv & k^{2} N_{2}(t, s)+i k\left(M_{2}(t, s)+\frac{\lambda}{2} g_{0}(t) N_{1}(t, s)\right) \\
& \left.+L_{2}(t, s)+\frac{\lambda}{2} g_{0}(t) M_{1}(t, s)\right)+\frac{\lambda}{4} g_{1}(t) N_{1}(t, s),
\end{aligned}
$$

where $L_{j}(t, s), M_{j}(t, s)$, and $N_{j}(t, s), j=1,2$ solve the Goursat problem for a system of linear differential equations with coefficients determined by $\left\{g_{l}(t)\right\}_{0}^{2}$ and with the boundary conditions

$$
\begin{aligned}
& L_{2}(t,-t)=M_{2}(t,-t)=N_{2}(t,-t)=0, \\
& L_{1}(t, t)=\lambda g_{0}^{3}(t)-\frac{1}{2} g_{2}(t), \\
& M_{1}(t, t)=g_{1}(t), \\
& N_{1}(t, t)=2 g_{0}(t) .
\end{aligned}
$$

Analogously, the functions $\mathcal{F}_{1}$ and $\mathcal{F}_{2}$ are expressed in terms of $\mathcal{L}_{j}, \mathcal{M}_{j}$, and $\mathcal{N}_{j}$ satisfying the similar system of equations, with $\left\{g_{l}(t)\right\}_{0}^{2}$ replaced by $\left\{h_{l}(t)\right\}_{0}^{2}$.

Similarly to the sG equation, the proof of the lemma is based on the Gelfand-Levitan-Marchenko representation for the solution of the $t$-equation in the Lax pair (1.7) with the initial condition $\mu(0, k)=I$ ( $x$ is fixed):

$$
\mu(t, k)=I+\int_{-t}^{t}\left(L(t, s)+i k M(t, s)+k^{2} N(t, s)\right) e^{4 i k^{3}(t-s) \sigma_{3}} d s .
$$

The functions $L_{j}, M_{j}$, and $N_{j}$ are simply expressed in terms of the matrix entries of $L, M$, and $N$. The details can be found in [9].

As in the case of the sG equation, we will continue the analysis of the global relation in the case $a(k) \equiv 1, b(k) \equiv 0$ corresponding to the zero initial conditions $q(x, 0)=0$. In this case, it follows from (2.36) that

$$
e^{2 i k L} A(t, k) \mathcal{B}(t, k)-B(t, k) \mathcal{A}(t, k)=e^{8 i k^{3} t} c(t, k), \quad k \in D_{1} \cup D_{3},
$$

where

$$
\begin{aligned}
c(t, k) & =O\left(\frac{1}{k}\right), & k \in D_{1}=\left\{k: \operatorname{Im} k>0, \operatorname{Im} k^{3}>0\right\}, \\
e^{-2 i k L} c(t, k) & =O\left(\frac{1}{k}\right), & k \in D_{3}=\left\{k: \operatorname{Im} k<0, \operatorname{Im} k^{3}>0\right\} .
\end{aligned}
$$

In the case of the mKdV I equation, the given (linearly well-posed) boundary conditions are $g_{0}(t)$, $h_{0}(t)$, and $h_{1}(t)$, and we are looking for expressions for $g_{1}(t), g_{2}(t)$, and $h_{2}(t)$.

Substitute (4.26) into (4.29) and rewrite the resulting equation in the form:

$$
\begin{aligned}
-e^{2 i k L} & \int_{0}^{t} \mathcal{F}_{1}^{(0)}(t, 2 \tau-t) e^{8 i k^{3} \tau} d \tau+\int_{0}^{t} F_{1}^{(0)}(t, 2 \tau-t) e^{8 i k^{3} \tau} d \tau \\
& +i k \int_{0}^{t} F_{1}^{(1)}(t, 2 \tau-t) e^{8 i k^{3} \tau} d \tau=G_{1}(t, k)+G_{2}(t, k)+e^{8 i k^{3} t} c(t, k),
\end{aligned}
$$


where

$$
\begin{aligned}
G_{1}(t, k)= & e^{2 i k L}\left(i k \int_{0}^{t} \mathcal{F}_{1}^{(1)}(t, 2 \tau-t) e^{8 i k^{3} \tau} d \tau+k^{2} \int_{0}^{t} \mathcal{F}_{1}^{(2)}(t, 2 \tau-t) e^{8 i k^{3} \tau} d \tau\right) \\
& -k^{2} \int_{0}^{t} F_{1}^{(2)}(t, 2 \tau-t) e^{8 i k^{3} \tau} d \tau, \\
G_{2}(t, k)= & 2 e^{2 i k L} \int_{0}^{t} \overline{F_{2}(t, t-2 \tau, k)} e^{8 i k^{3} \tau} d \tau \int_{0}^{t} \mathcal{F}_{1}(t, 2 \tau-t, k) e^{8 i k^{3} \tau} d \tau \\
& -2 \int_{0}^{t} \frac{\mathcal{F}_{2}(t, t-2 \tau, k)}{\mathcal{F}^{8 i k^{3}} \tau} d \tau \int_{0}^{t} F_{1}(t, 2 \tau-t, k) e^{8 i k^{3} \tau} d \tau .
\end{aligned}
$$

Let $D=\{k: 0<\arg k<\pi / 3\}$. Considering (4.31) in $D$ as well as replacing $k$ by $E k$ and by $E^{2} k$ in (4.31), where $E=e^{\frac{2 \pi i}{3}}$, we obtain three equations, which are valid for $k \in D$. These equations can be written in the vector form as follows:

$$
\mathcal{E}(k) U(t, k)=H_{1}(t, k)+H_{2}(t, k)+e^{8 i k^{3} t} H_{c}(t, k), \quad k \in D,
$$

where

$$
\begin{gathered}
\mathcal{E}(k)=\left(\begin{array}{ccc}
-e^{2 i k L} & 1 & 1 \\
-e^{2 i E k L} & 1 & E \\
-1 & e^{-2 i E^{2} k L} & E^{2} e^{-2 i E^{2} k L}
\end{array}\right), \\
U(t, k)= \\
\left(\int_{0}^{t} \mathcal{F}_{1}^{(0)}(t, 2 \tau-t) e^{8 i k^{3} \tau} d \tau, \int_{0}^{t} F_{1}^{(0)}(t, 2 \tau-t) e^{8 i k^{3} \tau} d \tau, i k \int_{0}^{t} F_{1}^{(1)}(t, 2 \tau-t) e^{8 i k^{3} \tau} d \tau\right)^{T}, \\
H_{j}(t, k)=\left(G_{j}(t, k), G_{j}(t, E k), e^{-2 i E^{2} k L} G_{j}\left(t, E^{2} k\right)\right)^{T}, \quad j=1,2, \\
H_{c}(t, k)=\left(c(t, k), c(t, E k), e^{-2 i E^{2} k L} c\left(t, E^{2} k\right)\right)^{T} .
\end{gathered}
$$

Notice that $\operatorname{det} \mathcal{E}(k) \rightarrow 1-E \neq 0$ as $|k| \rightarrow \infty, k \in \bar{D}$.

Multiply (4.34) by $\operatorname{diag}\left\{k^{2}, k^{2},-i k\right\} \mathcal{E}^{-1}(k) e^{-8 i k^{3} t^{\prime}}, 0<t^{\prime}<t$, and integrate over the contour $\partial D^{(0)}$, which is the boundary of $D$ deformed (in its finite part) to pass above the zeros of $\operatorname{det} \mathcal{E}(k)$. Then $(4.30)$ implies that the term containing $H_{c}$ vanishes.

In order to evaluate the other terms we will use the following identities (see, e.g., [8]):

$$
\begin{aligned}
\int_{\partial D^{(0)}} k^{2} \int_{0}^{t} \alpha(\tau) e^{8 i k^{3}\left(\tau-t^{\prime}\right)} d \tau d k & =\frac{\pi}{12} \alpha\left(t^{\prime}\right) \\
\int_{\partial D^{(0)}} k^{m} \int_{0}^{t} \alpha(\tau) e^{8 i k^{3}\left(\tau-t^{\prime}\right)} d \tau d k & =\int_{\partial D^{(0)}} k^{m}\left(\int_{0}^{t^{\prime}} \alpha(\tau) e^{8 i k^{3}\left(\tau-t^{\prime}\right)} d \tau-\frac{1}{8 i k^{3}} \alpha\left(t^{\prime}\right)\right) d k,
\end{aligned}
$$

where $m=3,4$ and $\alpha(\tau)$ is a smooth function for $0<\tau<t$. Then the integration by part together with Jordan's lemma show that one can pass to the limit as $t^{\prime} \rightarrow t$ in the right-hand side of (4.36).

Applying (4.36) to the integral term containing $H_{1}$ one obtains

$$
\begin{aligned}
& \int_{\partial D^{(0)}} \operatorname{diag}\left\{k^{2}, k^{2},-i k\right\} \mathcal{E}^{-1}(k) H_{1}(t, k) e^{-8 i k^{3} t^{\prime}} d k= \\
& \quad \int_{\partial D^{(0)}}\left(\begin{array}{ccc}
k^{2} & 0 & 0 \\
0 & k^{2} & 0 \\
0 & 0 & -i k
\end{array}\right) \mathcal{E}^{-1}(k)\left(\begin{array}{c}
\tilde{G}_{1}\left(t, t^{\prime}, k\right) \\
\tilde{G}_{1}\left(t, t^{\prime}, E k\right) \\
e^{-2 i E^{2} k L} \tilde{G}_{1}\left(t, t^{\prime}, E^{2} k\right)
\end{array}\right) d k
\end{aligned}
$$


where

$$
\begin{aligned}
\tilde{G}_{1}\left(t, t^{\prime}, k\right)= & e^{2 i k L}\left\{i k \int_{0}^{t^{\prime}} \mathcal{F}_{1}^{(1)}(t, 2 \tau-t) e^{8 i k^{3}\left(\tau-t^{\prime}\right)} d \tau-\frac{1}{8 k^{2}} \mathcal{F}_{1}^{(1)}\left(t, 2 t^{\prime}-t\right)\right. \\
& \left.+k^{2} \int_{0}^{t^{\prime}} \mathcal{F}_{1}^{(2)}(t, 2 \tau-t) e^{8 i k^{3}\left(\tau-t^{\prime}\right)} d \tau-\frac{1}{8 i k} \mathcal{F}_{1}^{(2)}\left(t, 2 t^{\prime}-t\right)\right\} \\
& -k^{2} \int_{0}^{t^{\prime}} F_{1}^{(2)}(t, 2 \tau-t) e^{8 i k^{3}\left(\tau-t^{\prime}\right)} d \tau+\frac{1}{8 i k} F_{1}^{(2)}\left(t, 2 t^{\prime}-t\right) .
\end{aligned}
$$

Applying (4.35) to the integral in the left-hand side of (4.34) we arrive at the equation

$$
\begin{aligned}
\left(\begin{array}{c}
\mathcal{F}_{1}^{(0)}\left(t, 2 t^{\prime}-t\right) \\
F_{1}^{(0)}\left(t, 2 t^{\prime}-t\right) \\
F_{1}^{(1)}\left(t, 2 t^{\prime}-t\right)
\end{array}\right)= & \frac{12}{\pi} \int_{\partial D^{(0)}}\left(\begin{array}{ccc}
k^{2} & 0 & 0 \\
0 & k^{2} & 0 \\
0 & 0 & -i k
\end{array}\right) \mathcal{E}^{-1}(k)\left(\begin{array}{c}
\tilde{G}_{1}\left(t, t^{\prime}, k\right) \\
\tilde{G}_{1}\left(t, t^{\prime}, E k\right) \\
e^{-2 i E^{2} k L} \tilde{G}_{1}\left(t, t^{\prime}, E^{2} k\right)
\end{array}\right) d k \\
& +\frac{12}{\pi} \int_{\partial D^{(0)}}\left(\begin{array}{ccc}
k^{2} & 0 & 0 \\
0 & k^{2} & 0 \\
0 & 0 & -i k
\end{array}\right) \mathcal{E}^{-1}(k)\left(\begin{array}{c}
G_{2}(t, k) \\
G_{2}(t, E k) \\
e^{-2 i E^{2} k L} G_{2}\left(t, E^{2} k\right)
\end{array}\right) e^{-8 i k^{3} t^{\prime}} d k
\end{aligned}
$$

Evaluating this equation at $t^{\prime}=t$ and using (4.27) and (4.28) we find the following equations for $h_{2}(t)$, $g_{1}(t)$, and $g_{2}(t)$ :

$$
\begin{aligned}
g_{1}(t)= & \frac{1}{2} g_{0}(t) N_{2}(t, t)-\frac{12 i}{\pi} \int_{\partial D^{(0)}} k\left[\mathcal{E}^{-1}(k)\right]_{3}\left(\begin{array}{c}
\tilde{G}_{1}(t, k) \\
\tilde{G}_{1}(t, E k) \\
e^{-2 i E^{2} k L} \tilde{G}_{1}\left(t, E^{2} k\right)
\end{array}\right) d k \\
& -\frac{12 i}{\pi} \int_{\partial D^{(0)}} k\left[\mathcal{E}^{-1}(k)\right]_{3}\left(\begin{array}{c}
G_{2}(t, k) \\
G_{2}(t, E k) \\
e^{-2 i E^{2} k L} G_{2}\left(t, E^{2} k\right)
\end{array}\right) e^{-8 i k^{3} t} d k, \\
g_{2}(t)= & 2 \lambda g_{0}^{3}(t)+g_{0}(t) M_{2}(t, t)+\frac{1}{2} g_{1}(t) N_{2}(t, t)-\frac{24}{\pi} \int_{\partial D^{(0)}} k^{2}\left[\mathcal{E}^{-1}(k)\right]_{2}\left(\begin{array}{c}
\tilde{G}_{1}(t, k) \\
\tilde{G}_{1}(t, E k) \\
e^{-2 i E^{2} k L} \tilde{G}_{1}\left(t, E^{2} k\right)
\end{array}\right) d k \\
& -\frac{24}{\pi} \int_{\partial D^{(0)}} k^{2}\left[\mathcal{E}^{-1}(k)\right]_{2}\left(\begin{array}{c}
G_{2}(t, k) \\
G_{2}(t, E k) \\
e^{-2 i E^{2} k L} G_{2}\left(t, E^{2} k\right)
\end{array}\right) e^{-8 i k^{3} t} d k, \\
h_{2}(t)= & 2 \lambda h_{0}^{3}(t)+h_{0}(t) \mathcal{M}_{2}(t, t)+\frac{1}{2} h_{1}(t) \mathcal{N}_{2}(t, t)-\frac{24}{\pi} \int_{\partial D^{(0)}} k^{2}\left[\mathcal{E}^{-1}(k)\right]_{1}\left(\begin{array}{c}
\tilde{G}_{1}(t, k) \\
\tilde{G}_{1}(t, E k) \\
e^{-2 i E^{2} k L} \tilde{G}_{1}\left(t, E^{2} k\right)
\end{array}\right) d k \\
& -\frac{24}{\pi} \int_{\partial D^{(0)}} k^{2}\left[\mathcal{E}^{-1}(k)\right]_{1}\left(\begin{array}{c}
G_{2}(t, k) \\
G_{2}(t, E k) \\
e^{-2 i E^{2} k L} G_{2}\left(t, E^{2} k\right)
\end{array}\right) e^{-8 i k^{3} t} d k,
\end{aligned}
$$

where $\left[\mathcal{E}^{-1}(k)\right]_{j}, j=1,2,3$, denotes the $j^{\text {th }}$ row of $\mathcal{E}^{-1}(k)$ and

$$
\begin{aligned}
\tilde{G}_{1}(t, k)= & e^{2 i k L}\left\{i k \int_{0}^{t}\left(\mathcal{M}_{1}(t, 2 \tau-t)-\frac{1}{2} h_{0}(t) \mathcal{N}_{2}(t, 2 \tau-t)\right) e^{8 i k^{3}(\tau-t)} d \tau-\frac{1}{8 k^{2}} h_{1}(t)\right. \\
& \left.+\frac{1}{16 k^{2}} h_{0}(t) \mathcal{N}_{2}(t, t)+k^{2} \int_{0}^{t} \mathcal{N}_{1}(t, 2 \tau-t) e^{8 i k^{3}(\tau-t)} d \tau-\frac{1}{4 i k} h_{0}(t)\right\} \\
& -k^{2} \int_{0}^{t} N_{1}(t, 2 \tau-t) e^{8 i k^{3}(\tau-t)} d \tau+\frac{1}{4 i k} g_{0}(t) .
\end{aligned}
$$


The functions $\tilde{G}_{1}(t, k), G_{2}(t, k), N_{2}(t, t), M_{2}(t, t), \mathcal{N}_{2}(t, t)$, and $\mathcal{M}_{2}(t, t)$ involved in (4.40) can be expressed in terms of $\Phi(t, k)$ and $\Psi(t, k)$, see Definitions 2 and 3. Indeed, (3.6) and (3.9) together with (4.2) give

$$
\begin{aligned}
\int_{0}^{t} F_{1}(t, 2 \tau-t, k) e^{8 i k^{3} \tau} d \tau & =\frac{1}{2} \Phi_{1}(t, k) e^{8 i k^{3} t}, \\
\int_{0}^{t} \mathcal{F}_{1}(t, 2 \tau-t, k) e^{8 i k^{3} \tau} d \tau & =\frac{1}{2} \Psi_{1}(t, k) e^{8 i k^{3} t}, \\
\int_{0}^{t} \overline{F_{2}(t, t-2 \tau, \bar{k})} e^{8 i k^{3} \tau} d \tau & =\frac{1}{2}\left(\overline{\Phi_{2}(t, \bar{k})}-1\right), \\
\int_{0}^{t} \overline{\mathcal{F}_{2}(t, t-2 \tau, \bar{k})} e^{8 i k^{3} \tau} d \tau & =\frac{1}{2}\left(\overline{\Psi_{2}(t, \bar{k})}-1\right) .
\end{aligned}
$$

Hence, $G_{2}(t, k)$ can be written as follows:

$$
G_{2}(t, k)=\frac{1}{2} e^{8 i k^{3} t}\left\{e^{2 i k L}\left(\overline{\Phi_{2}(t, \bar{k})}-1\right) \Psi_{1}(t, k)-\left(\overline{\Psi_{2}(t, \bar{k})}-1\right) \Phi_{1}(t, k)\right\} .
$$

Since the exponentials in (4.42) depend on $k$ only through $k^{3}$, supplementing (4.42) with the two equations obtained from (4.42) by replacing $k$ with $E k$ and $E^{2} k$ and taking into account (4.27) we obtain a linear system of equations, the solution of which gives

$$
\left(\begin{array}{c}
k^{2} \int_{0}^{t} F_{1}^{(2)}(t, 2 \tau-t, k) e^{8 i k^{3} \tau} d \tau \\
i k \int_{0}^{t} F_{1}^{(1)}(t, 2 \tau-t, k) e^{8 i k^{3} \tau} d \tau \\
\int_{0}^{t} F_{1}^{(0)}(t, 2 \tau-t, k) e^{8 i k^{3} \tau} d \tau
\end{array}\right)=\frac{1}{6} e^{8 i k^{3} t}\left(\begin{array}{c}
\Phi_{1}(t, k)+E \Phi_{1}(t, E k)+E^{2} \Phi_{1}\left(t, E^{2} k\right) \\
\Phi_{1}(t, k)+E^{2} \Phi_{1}(t, E k)+E \Phi_{1}\left(t, E^{2} k\right) \\
\Phi_{1}(t, k)+\Phi_{1}(t, E k)+\Phi_{1}\left(t, E^{2} k\right)
\end{array}\right)
$$

Similarly, supplementing (4.44) with the two equations obtained from (4.44) by replacing $k$ with $E k$ and $E^{2} k$ and taking into account (4.27) we find

$$
\left(\begin{array}{c}
k^{2} \int_{0}^{t} F_{2}^{(2)}(t, t-2 \tau, k) e^{8 i k^{3} \tau} d \tau \\
i k \int_{0}^{t} F_{2}^{(1)}(t, t-2 \tau, k) e^{8 i k^{3} \tau} d \tau \\
\int_{0}^{t} F_{2}^{(0)}(t, t-2 \tau, k) e^{8 i k^{3} \tau} d \tau
\end{array}\right)=\frac{1}{6}\left(\begin{array}{c}
\Phi_{2}(t, k)+E \Phi_{2}(t, E k)+E^{2} \Phi_{2}\left(t, E^{2} k\right) \\
\Phi_{2}(t, k)+E^{2} \Phi_{2}(t, E k)+E \Phi_{2}\left(t, E^{2} k\right) \\
\Phi_{1}(t, k)+\Phi_{1}(t, E k)+\Phi_{1}\left(t, E^{2} k\right)-3
\end{array}\right)
$$

Using (4.35) and the expressions for $F_{2}^{(1)}(t, s)$ and $F_{2}^{(2)}(t, s)$ in terms of $N_{j}(t, s), j=1,2$ and $M_{2}(t, s)$, see (4.27), from (4.48) we conclude that

$$
\begin{aligned}
& N_{2}(t, t)=\frac{2}{\pi} \int_{\partial D^{(0)}}\left(\Phi_{2}(t, k)+E \Phi_{2}(t, E k)+E^{2} \Phi_{2}\left(t, E^{2} k\right)\right) d k \\
& M_{2}(t, t)=-\lambda g_{0}^{2}(t)-\frac{2 i}{\pi} \int_{\partial D^{(0)}} k\left(\Phi_{2}(t, k)+E^{2} \Phi_{2}(t, E k)+E \Phi_{2}\left(t, E^{2} k\right)\right) d k .
\end{aligned}
$$

Similarly,

$$
\begin{aligned}
\mathcal{N}_{2}(t, t) & =\frac{2}{\pi} \int_{\partial D^{(0)}}\left(\Psi_{2}(t, k)+E \Psi_{2}(t, E k)+E^{2} \Psi_{2}\left(t, E^{2} k\right)\right) d k \\
\mathcal{M}_{2}(t, t) & =-\lambda g_{0}^{2}(t)-\frac{2 i}{\pi} \int_{\partial D^{(0)}} k\left(\Psi_{2}(t, k)+E^{2} \Psi_{2}(t, E k)+E \Psi_{2}\left(t, E^{2} k\right)\right) d k .
\end{aligned}
$$


Substituting (4.47) and (4.50) into (4.41) we obtain the following expression for $\tilde{G}_{1}$ :

$$
\begin{aligned}
\tilde{G}_{1}(t, k)= & e^{2 i k L}\left\{\frac{1}{3} e^{8 i k^{3} t}\left[\Psi_{1}(t, k)-\Psi_{1}(t, E k)-\Psi_{1}\left(t, E^{2} k\right)\right]-\frac{1}{8 k^{2}} h_{1}(t)-\frac{1}{4 i k} h_{0}(t)\right. \\
& \left.+\frac{1}{8 \pi k^{2}} h_{0}(t) \int_{\partial D^{(0)}}\left(\Psi_{2}(t, \zeta)+E \Psi_{2}(t, E \zeta)+E^{2} \Psi_{2}\left(t, E^{2} \zeta\right)\right) d \zeta\right\} \\
& -\frac{1}{6} e^{8 i k^{3} t}\left[\Phi_{1}(t, k)+E \Phi_{1}(t, E k)+E^{2} \Phi_{1}\left(t, E^{2} k\right)\right]+\frac{1}{4 i k} g_{0}(t) .
\end{aligned}
$$

Using (4.46), (4.51), (4.49), and (4.50) in (4.40) we obtain the equations for $g_{1}, g_{2}$, and $h_{2}$ in terms of $\Phi$ and $\Psi$. These equations, together with (3.5) and the similar equation for $\Psi(t, k)$ constitute a system of four nonlinear ODEs for $\Phi_{1}, \Phi_{2}, \Psi_{1}$, and $\Psi_{2}$.

\section{mKdV II:}

The integral representations for $A$ and $B$ are the same as in (4.26)- (4.28) but with $g_{0}$ and $g_{2}$ replaced by $-g_{0}$ and $-g_{2}$, respectively. Similarly, the integral representations for $\mathcal{A}$ and $\mathcal{B}$ are the same as in the case of the mKdV I, with $h_{0}$ and $h_{2}$ replaced by $-h_{0}$ and $-h_{2}$, respectively.

The global relation (4.29) and relations (4.30) become

$$
e^{-2 i k L} A(t, k) \mathcal{B}(t, k)-B(t, k) \mathcal{A}(t, k)=e^{8 i k^{3} t} c(t, k), \quad k \in D_{1} \cup D_{3},
$$

and

$$
\begin{aligned}
c(t, k)=O\left(\frac{1}{k}\right), & k \in D_{1}=\left\{k: \operatorname{Im} k<0, \operatorname{Im} k^{3}>0\right\}, \\
e^{2 i k L} c(t, k)=O\left(\frac{1}{k}\right), & k \in D_{3}=\left\{k: \operatorname{Im} k>0, \operatorname{Im} k^{3}>0\right\},
\end{aligned}
$$

respectively.

Let $g_{0}(t), g_{1}(t)$, and $h_{0}(t)$ be the given boundary conditions. Then the analysis of the global relation consists in finding equations for $g_{2}, h_{1}$, and $h_{2}$. Substitute (4.26) into (4.52) and rewrite the resulting equation in the form:

$$
\begin{gathered}
-e^{-2 i k L} \int_{0}^{t} \mathcal{F}_{1}^{(0)}(t, 2 \tau-t) e^{8 i k^{3} \tau} d \tau-e^{-2 i k L} i k \int_{0}^{t} \mathcal{F}_{1}^{(1)}(t, 2 \tau-t) e^{8 i k^{3} \tau} d \tau \\
+\int_{0}^{t} F_{1}^{(0)}(t, 2 \tau-t) e^{8 i k^{3} \tau} d \tau=G_{1}(t, k)+G_{2}(t, k)+e^{8 i k^{3} t} c(t, k),
\end{gathered}
$$

where

$$
\begin{aligned}
G_{1}(t, k)= & e^{-2 i k L} k^{2} \int_{0}^{t} \mathcal{F}_{1}^{(2)}(t, 2 \tau-t) e^{8 i k^{3} \tau} d \tau-i k \int_{0}^{t} F_{1}^{(1)}(t, 2 \tau-t) e^{8 i k^{3} \tau} d \tau \\
& -k^{2} \int_{0}^{t} F_{1}^{(2)}(t, 2 \tau-t) e^{8 i k^{3} \tau} d \tau \\
G_{2}(t, k)= & 2 e^{-2 i k L} \int_{0}^{t} \overline{F_{2}(t, t-2 \tau, k)} e^{8 i k^{3} \tau} d \tau \int_{0}^{t} \mathcal{F}_{1}(t, 2 \tau-t, k) e^{8 i k^{3} \tau} d \tau \\
& -2 \int_{0}^{t} \frac{\mathcal{F}_{2}(t, t-2 \tau, k)}{8 i k^{3} \tau} d \tau \int_{0}^{t} F_{1}(t, 2 \tau-t, k) e^{8 i k^{3} \tau} d \tau .
\end{aligned}
$$

Let, as above, $D=\{k: 0<\arg k<\pi / 3\}$. Considering (4.54) in $D$ as well as replacing $k$ by $E k$ and by $E^{2} k$ in (4.54), where $E=e^{\frac{2 \pi i}{3}}$, we get three equations valid for $k \in D$, which can be written in the vector form as follows:

$$
\mathcal{E}(k) U(t, k)=H_{1}(t, k)+H_{2}(t, k)+e^{8 i k^{3} t} H_{c}(t, k), \quad k \in D,
$$


where

$$
\mathcal{E}(k)=\left(\begin{array}{ccc}
-1 & -1 & e^{2 i k L} \\
-1 & -E & e^{2 i E k L} \\
-e^{-2 i E^{2} k L} & -E^{2} e^{-2 i E^{2} k L} & 1
\end{array}\right)
$$

$$
\begin{gathered}
U(t, k)= \\
\left(\int_{0}^{t} \mathcal{F}_{1}^{(0)}(t, 2 \tau-t) e^{8 i k^{3} \tau} d \tau, i k \int_{0}^{t} \mathcal{F}_{1}^{(1)}(t, 2 \tau-t) e^{8 i k^{3} \tau} d \tau, \int_{0}^{t} F_{1}^{(0)}(t, 2 \tau-t) e^{8 i k^{3} \tau} d \tau\right)^{T}, \\
H_{j}(t, k)=\left(e^{2 i k L} G_{j}(t, k), e^{2 i E k L} G_{j}(t, E k), G_{j}\left(t, E^{2} k\right)\right)^{T}, \quad j=1,2 \\
H_{c}(t, k)=\left(e^{2 i k L} c(t, k), e^{2 i E k L} c(t, E k), c\left(t, E^{2} k\right)\right)^{T}
\end{gathered}
$$

Notice that $\operatorname{det} \mathcal{E}(k) \rightarrow E-1 \neq 0$ as $|k| \rightarrow \infty, k \in \bar{D}$.

Multiply (4.57) by $\operatorname{diag}\left\{k^{2},-i k, k^{2}\right\} \mathcal{E}^{-1}(k) e^{-8 i k^{3} t^{\prime}}, 0<t^{\prime}<t$, and integrate over the contour $\partial D^{(0)}$, which is the boundary of $D$ deformed (in its finite part) to pass above the zeros of $\operatorname{det} \mathcal{E}(k)$. Then $(4.53)$ implies that the term containing $H_{c}$ vanishes, and the resulting equation takes the form

$$
\begin{aligned}
\left(\begin{array}{c}
\mathcal{F}_{1}^{(0)}\left(t, 2 t^{\prime}-t\right) \\
\mathcal{F}_{1}^{(1)}\left(t, 2 t^{\prime}-t\right) \\
F_{1}^{(0)}\left(t, 2 t^{\prime}-t\right)
\end{array}\right)= & \frac{12}{\pi} \int_{\partial D^{(0)}}\left(\begin{array}{ccc}
k^{2} & 0 & 0 \\
0 & k^{2} & 0 \\
0 & 0 & -i k
\end{array}\right) \mathcal{E}^{-1}(k)\left(\begin{array}{c}
e^{2 i k L} \tilde{G}_{1}\left(t, t^{\prime}, k\right) \\
e^{2 i E k L} \tilde{G}_{1}\left(t, t^{\prime}, E k\right) \\
\tilde{G}_{1}\left(t, t^{\prime}, E^{2} k\right)
\end{array}\right) d k \\
& +\frac{12}{\pi} \int_{\partial D^{(0)}}\left(\begin{array}{ccc}
k^{2} & 0 & 0 \\
0 & k^{2} & 0 \\
0 & 0 & -i k
\end{array}\right) \mathcal{E}^{-1}(k)\left(\begin{array}{c}
e^{2 i k L} G_{2}(t, k) \\
e^{2 i E k L} G_{2}(t, E k) \\
G_{2}\left(t, E^{2} k\right)
\end{array}\right) e^{-8 i k^{3} t^{\prime}} d k
\end{aligned}
$$

where

$$
\begin{aligned}
\tilde{G}_{1}\left(t, t^{\prime}, k\right)= & e^{-2 i k L}\left\{k^{2} \int_{0}^{t^{\prime}} \mathcal{F}_{1}^{(2)}(t, 2 \tau-t) e^{8 i k^{3}\left(\tau-t^{\prime}\right)} d \tau-\frac{1}{8 i k} \mathcal{F}_{1}^{(2)}\left(t, 2 t^{\prime}-t\right)\right\} \\
& -i k \int_{0}^{t^{\prime}} F_{1}^{(1)}(t, 2 \tau-t) e^{8 i k^{3}\left(\tau-t^{\prime}\right)} d \tau+\frac{1}{8 k^{2}} F_{1}^{(1)}\left(t, 2 t^{\prime}-t\right) \\
& -k^{2} \int_{0}^{t^{\prime}} F_{1}^{(2)}(t, 2 \tau-t) e^{8 i k^{3}\left(\tau-t^{\prime}\right)} d \tau+\frac{1}{8 i k} F_{1}^{(2)}\left(t, 2 t^{\prime}-t\right) .
\end{aligned}
$$

Evaluating this equation at $t^{\prime}=t$ and using (4.27) and (4.28) we find the following equations for $g_{2}(t)$, 
$h_{1}(t)$, and $h_{2}(t)$ :

$$
\begin{aligned}
g_{1}(t)= & -\frac{1}{2} g_{0}(t) N_{2}(t, t)-\frac{12 i}{\pi} \int_{\partial D^{(0)}} k\left[\mathcal{E}^{-1}(k)\right]_{3}\left(\begin{array}{c}
e^{2 i k L} \tilde{G}_{1}(t, k) \\
e^{2 i E k L} \tilde{G}_{1}(t, E k) \\
\tilde{G}_{1}\left(t, E^{2} k\right)
\end{array}\right) \\
& -\frac{12 i}{\pi} \int_{\partial D^{(0)}} k\left[\mathcal{E}^{-1}(k)\right]_{2}\left(\begin{array}{c}
e^{2 i k L} G_{2}(t, k) \\
e^{2 i E k L} G_{2}(t, E k) \\
G_{2}\left(t, E^{2} k\right)
\end{array}\right) e^{-8 i k^{3} t} d k, \\
h_{1}(t)= & -\frac{1}{2} h_{0}(t) N_{2}(t, t)-\frac{12 i}{\pi} \int_{\partial D^{(0)}} k\left[\mathcal{E}^{-1}(k)\right]_{2}\left(\begin{array}{c}
e^{2 i k L} \tilde{G}_{1}(t, k) \\
e^{2 i E k L} \tilde{G}_{1}(t, E k) \\
\tilde{G}_{1}\left(t, E^{2} k\right)
\end{array}\right) d k \\
& -\frac{12 i}{\pi} \int_{\partial D^{(0)}} k\left[\mathcal{E}^{-1}(k)\right]_{3}\left(\begin{array}{c}
e^{2 i k L} G_{2}(t, k) \\
e^{2 i E k L} G_{2}(t, E k) \\
G_{2}\left(t, E^{2} k\right)
\end{array}\right) e^{-8 i k^{3} t} d k, \\
h_{2}(t)= & 2 \lambda h_{0}^{3}(t)+h_{0}(t) \mathcal{M}_{2}(t, t)-\frac{1}{2} h_{1}(t) \mathcal{N}_{2}(t, t)+\frac{24}{\pi} \int_{\partial D^{(0)}} k^{2}\left[\mathcal{E}^{-1}(k)\right]_{1}\left(\begin{array}{c}
e^{2 i k L} \tilde{G}_{1}(t, k) \\
e^{2 i E k L} \tilde{G}_{1}(t, E k) \\
\tilde{G}_{1}\left(t, E^{2} k\right)
\end{array}\right) d k \\
& +\frac{24}{\pi} \int_{\partial D^{(0)}} k^{2}\left[\mathcal{E}^{-1}(k)\right]_{1}\left(\begin{array}{c}
e^{2 i k L} G_{2}(t, k) \\
e^{2 i E k L} G_{2}(t, E k) \\
G_{2}\left(t, E^{2} k\right)
\end{array}\right) e^{-8 i k^{3} t} d k,
\end{aligned}
$$

where

$$
\begin{aligned}
\tilde{G}_{1}(t, k)= & e^{-2 i k L}\left\{k^{2} \int_{0}^{t} \mathcal{N}_{1}(t, 2 \tau-t) e^{8 i k^{3}(\tau-t)} d \tau+\frac{1}{4 i k} h_{0}(t)\right\} \\
& -i k \int_{0}^{t}\left(M_{1}(t, 2 \tau-t)+\frac{1}{2} g_{0}(t) N_{2}(t, 2 \tau-t)\right) e^{8 i k^{3}(\tau-t)} d \tau+\frac{1}{8 k^{2}} g_{1}(t) \\
& +\frac{1}{16 k^{2}} g_{0}(t) N_{2}(t, t)-k^{2} \int_{0}^{t} N_{1}(t, 2 \tau-t) e^{8 i k^{3}(\tau-t)} d \tau-\frac{1}{4 i k} g_{0}(t) .
\end{aligned}
$$

Now one can express the functions involved in (4.60) in terms of $\Phi$ and $\Psi$. The formulas for $N_{2}(t, t)$, $M_{2}(t, t), \mathcal{N}_{2}(t, t)$, and $\mathcal{M}_{2}(t, t)$ have the same form as in the case of mKdV I, (4.49) and (4.50), whereas

$$
G_{2}(t, k)=\frac{1}{2} e^{8 i k^{3} t}\left\{e^{-2 i k L}\left(\overline{\Phi_{2}(t, \bar{k})}-1\right) \Psi_{1}(t, k)-\left(\overline{\Psi_{2}(t, \bar{k})}-1\right) \Phi_{1}(t, k)\right\}
$$

and

$$
\begin{aligned}
\tilde{G}_{1}(t, k)= & e^{-2 i k L}\left\{\frac{1}{6} e^{8 i k^{3} t}\left[\Psi_{1}(t, k)+E \Psi_{1}(t, E k)+E^{2} \Psi_{1}\left(t, E^{2} k\right)\right]+\frac{1}{4 i k} h_{0}(t)\right\} \\
& -\frac{1}{3} e^{8 i k^{3} t}\left[\Phi_{1}(t, k)-\Phi_{1}(t, E k)-\Phi_{1}\left(t, E^{2} k\right)\right]+\frac{1}{8 k^{2}} g_{1}(t)-\frac{1}{4 i k} g_{0}(t) \\
& +\frac{1}{8 \pi k^{2}} g_{0}(t) \int_{\partial D^{(0)}}\left(\Phi_{2}(t, \zeta)+E \Phi_{2}(t, E \zeta)+E^{2} \Phi_{2}\left(t, E^{2} \zeta\right)\right) d \zeta
\end{aligned}
$$

\section{Conclusions}

We have presented a general method for the analysis of initial boundary value problems for nonlinear integrable evolution equations on the finite interval and have applied this method to the sine-Gordon and the two mKdV equations. In particular:

1. Given the Dirichlet data for the sG equation, $q(0, t)=g_{0}(t)$ and $q(L, t)=g_{1}(t)$, we have characterized the Neumann boundary values $q_{x}(0, t)=g_{1}(t)$ and $q_{x}(L, t)=h_{1}(t)$ through a system of 
nonlinear ODEs for the functions $\Phi_{1}, \Phi_{2}, \Psi_{1}$, and $\Psi_{2}$. The functions $\Phi_{1}$ and $\Phi_{2}$ satisfy equations (3.5), the functions $\Psi_{1}$ and $\Psi_{2}$ satisfy similar equations, and the Neumann boundary values are given by equations (4.21) and (4.25).

Similarly, given the boundary data $q(0, t)=g_{0}(t), q(L, t)=h_{0}(t), q_{x}(L, t)=h_{1}(t)$ for the mKdV I equation $\left(q(0, t)=g_{0}(t), q_{x}(0, t)=g_{1}(t), q(L, t)=h_{0}(t)\right.$ for the mKdV II equation), we have characterized the boundary values $q_{x}(0, t)=g_{1}(t), q_{x x}(0, t)=g_{2}(t), q_{x x}(L, t)=h_{2}(t)\left(q_{x x}(0, t)=\right.$ $g_{2}(t), q_{x}(L, t)=h_{1}(t), q_{x x}(L, t)=h_{2}(t)$, respectively) through a system of nonlinear ODEs.

2. Given the initial conditions $q(x, 0)=q_{0}(x)\left(q(x, 0)=q_{0}(x)\right.$ and $q_{t}(x, 0)=q_{1}(x)$ for the sG equation $)$ we have defined $\{a(k), b(k)\}$, see Definition 1. Given $\left\{g_{l}(t)\right\}_{0}^{n-1}$ we have defined $\{A(k), B(k)\}$, and given $\left\{h_{l}(t)\right\}_{0}^{n-1}$ we have defined $\{\mathcal{A}(k), \mathcal{B}(k)\}$, see Definitions 2 and 3 .

3. Given $\{a(k), b(k), A(k), B(k), \mathcal{A}(k), \mathcal{B}(k)\}$ we have defined a Riemann-Hilbert problem for $M(x, t, k)$ and then we have defined $q(x, t)$ in terms of $M$. We have shown that $q(x, t)$ solves the given nonlinear equation and that

$$
\begin{aligned}
q(x, 0) & =q_{0}(x) \quad\left(\text { and } q_{t}(x, 0)=q_{1}(x) \text { for } \mathrm{sG}\right) \\
\partial_{x}^{l} q(0, t) & =g_{l}(t), \quad \partial_{x}^{l} q(L, t)=h_{l}(t), \quad 0 \leq l \leq n-1,
\end{aligned}
$$

see Theorem 1.

The most difficult step of this method is the analysis of the global relation coupling the spectral functions. Generally, this leads to a system of nonlinear ODEs. For integrable evolution PDEs on the half-line, there exist particular boundary conditions, the so-called linearizable boundary conditions, for which this nonlinear system can be avoided: the global relation yields directly $S(k)$ in terms of $s(k)$ and the prescribed boundary conditions $[5,6,7]$. Different aspects of linearizable boundary conditions have been studied by a number of authors, see, for example, [16]-[19]. The analysis of linearizable boundary conditions on a finite domain will be presented elsewhere. Here we only note that $x$-periodic boundary conditions belong to the linearizable class. In this case $S(k)=S_{L}(k)$ and the global relation simplifies. The analysis of this simplified global relation, together with the results presented in this paper, yields a new formalism for the solution of this classical problem.

The main advantage of the inverse scattering method, in comparison with the standard PDE techniques, is that it yields explicit asymptotic results. Indeed, using the inverse scattering method, the solution of the Cauchy problem on the line for an integrable nonlinear PDE can be expressed through the solution of a matrix Riemann-Hilbert problem which has a jump matrix involving an exponential $(x, t)$-dependence. By making use of the Deift-Zhou method [20] (which is a nonlinear version of the classical steepest descent method), it is possible to compute explicitly the long time behavior of the solution. Furthermore, using a nontrivial extension of the Deift-Zhou method [21], it is also possible to compute the small dispersion limit of the solution. Neither of these two important asymptotic results can be obtained by standard PDE techniques.

An important feature of the method of [3] is that it yields the solution of the given initial boundary value problem in terms of a matrix Riemann-Hilbert problem which also involves a jump matrix with an exponential $(x, t)$-dependence. The curve along which this jump matrix is defined, is now more complicated, but this does not pose any additional difficulties. Thus, it is again possible to obtain explicit asymptotic results. Indeed, for problems on the half-line, the long time asymptotics for decreasing and for time-periodic boundary conditions is obtained in [22]-[25] (see also [5] and [7]). Furthermore, the zero dispersion limit of the NLS equation is computed in [26]. For problems on the interval, it is again possible to study the asymptotic properties of the solution. In particular, it should be possible to study the small dispersion limit.

Another important feature of the method of [3] is that it characterizes the generalized Dirichlet-toNeumann map. For example, for the Dirichlet problem for the NLS equation on the half-line, the method of [3] yields $q_{x}(0, t)$ in terms of $q(x, 0)$ and $q(0, t)$. Actually, it is shown in [8] and [9] that $q_{x}(0, t)$ can be expressed explicitly through the solution of a system of nonlinear ODEs uniquely defined in terms of $q(x, 0)$ and $q(0, t)$. This is the first time in the literature that such an explicit result is obtained for a nonlinear evolution PDE. In this paper we have presented similar results for initial boundary problems on the interval. For example, for the case of the Dirichlet problem for the sG equation, equations (4.21) 
and (4.25) express $q_{x}(0, t)$ and $q_{x}(L, t)$ in terms of a system of four nonlinear ODEs which is uniquely defined in terms of $q(x, 0), q_{t}(x, 0), q(0, t)$, and $q(L, t)$. Such explicit results cannot be obtained by the standard PDE techniques.

\section{References}

[1] P.D.Lax, Integrals of nonlinear equations of evolution and solitary waves, Comm. Pure Appl. Math. 21 (1968), 467-490.

[2] G.S.Gardner, J.M.Green, M.D.Kruskal, and R.M.Miura, Phys. Rev. Lett. 19 (1967), 1095

[3] A.S.Fokas, A unified transform method for solving linear and certain nonlinear PDEs, Proc. R. Soc. London Ser. A, 453 (1997), 1411-1443

[4] A.S.Fokas, On the integrability of linear and nonlinear partial differential equations, J. Math. Phys., 41 (2000), 4188-4237

[5] A.S.Fokas, A.R.Its, and L.-Y.Sung, The nonlinear Schrödinger equation on the half-line, preprint

[6] A.S.Fokas, Integrable nonlinear evolution equations on the half-line, Commun. Math. Phys., 230 (2002), 1-39

[7] A.Boutet de Monvel, A.S.Fokas, and D.Shepelsky, The mKdV equation on the half-line, Journal of the Inst. of Math. Jussieu, 3 (2004), 139-164.

[8] A.Boutet de Monvel, A.S.Fokas, and D.Shepelsky, Analysis of the global relation for the nonlinear Schrödinger equation on the half-line, Lett. Math. Phys., 65 (2003), 199-212.

[9] A.S.Fokas, The generalized Dirichlet to Neumann map for certain nonlinear evolution PDEs, Comm. Pure Applied Math., in press.

[10] A.Boutet de Monvel and D.Shepelsky, The modified KdV equation on a finite interval, C. R. Math. Acad. Sci. Paris 337 (2003), 517-522.

[11] A.S.Fokas and A.R.Its, The nonlinear Schrödinger equation on the interval, J. Phys. A: Math. Gen. (2004)

[12] A.S.Fokas, Two dimensional linear PDEs in a convex polygon, Proc. R. Soc. London Ser.A, 457 (2001), 371-393

[13] X.Zhou, Inverse scattering transform for systems with rational spectral dependence, J. Differential Equations 115 (1995), 277-303.

[14] X.Zhou, The Riemann-Hilbert problem and inverse scattering, SIAM J. Math. Anal., 20 (1989), 966-986

[15] V.E.Zakharov and A.B.Shabat, A scheme for integrating the nonlinear equations of mathematical physics by the method of the inverse scattering problem. I and II, Funct. Anal. Appl. 8 (1974), 226-235 and 13 (1979), 166-174.

[16] E.K.Sklyanin, Boundary conditions for integrable equations, Funct.Anal.Appl. 21 (1987), 86-87

[17] V.O.Tarasov, An initial-boundary value problem for the nonlinear Schrödinger equation, Zap.Nauchn.Sem.LOMI 169 (1988), 151-165

[18] I.T.Habibullin, Bäcklund transformations and integrable initial-boundary value problems, in: Nonlinear and Turbulent Processes, 1, World Scientific, Singapore, 1990, 130-138

[19] V.E.Adler, B.Gürel, M.Gürses, and I.Habibullin, Boundary conditions for integrable equations, J.Phys. A, 30 (1997), 3500-3513 
[20] P.Deift and X.Zhou, A steepest descent method for oscillatory Riemann-Hilbert problems. Asymptotics for the MKdV equation, Ann. of Math. (2) 137 (1993), 295-368.

[21] P.Deift, S.Venakides, and X.Zhou, New results in small dispersion KdV by an extension of the steepest descent method for Riemann-Hilbert problems, Internat. Math. Res. Notices (1997), 286299.

[22] A.S.Fokas and A.R.Its, An initial-boundary value problem for the sine-Gordon equation, Theor. and Math. Physics, 92 (1992), 388-403.

[23] A.S.Fokas and A.R.Its, An initial-boundary value problem for the Korteweg-de Vries equation, Math. Comput. Simul., 37 (1994), 293-321.

[24] A.S.Fokas and A.R.Its, The linearization of the initial-boundary value problem of the nonlinear Schrödinger equation, SIAM J. Math. Anal., 27 (1996), 738-764

[25] A. Boutet de Monvel and V.Kotlyarov, Generation of asymptotic solitons of the nonlinear Schrödinger equation by boundary data, J. Math. Phys., 44 (2003), 3185-3215.

[26] S.Kamvissis, Semiclassical nonlinear Schrödinger on the half line, J. Math. Phys. 44, (2003), 58495868 . 